\title{
The Duration of Star Formation in Galactic Giant Molecular Clouds. I. The Great Nebula in Carina
}

\author{
Matthew S. Povich ${ }^{1,4}$ (D), Jessica T. Maldonado ${ }^{1,2}$, Evan Haze Nuñez ${ }^{1}$, and Thomas P. Robitaille ${ }^{3}$ (D) \\ ${ }^{1}$ Department of Physics and Astronomy, California State Polytechnic University, 3801 West Temple Ave, Pomona, CA 91768, USA; mspovich@cpp.edu \\ ${ }^{2}$ Department of Physics and Astronomy, Michigan State University, East Lansing, MI 48824, USA \\ ${ }^{3}$ Freelance Consultant, Headingley Enterprise and Arts Centre, Bennett Road Headingley, Leeds LS6 3HN, UK \\ Received 2019 April 19; revised 2019 May 29; accepted 2019 June 2; published 2019 August 9
}

\begin{abstract}
We present a novel infrared spectral energy distribution (SED) modeling methodology that uses likelihood-based weighting of the model fitting results to construct probabilistic Hertzsprung-Russell diagrams (pHRD) for X-rayidentified, intermediate-mass $\left(2-8 M_{\odot}\right)$, pre-main-sequence young stellar populations. This methodology is designed specifically for application to young stellar populations suffering strong, differential extinction ( $\Delta A_{V}>10 \mathrm{mag}$ ), typical of Galactic massive star-forming regions. We pilot this technique in the Carina Nebula Complex (CNC) by modeling the 1-8 $\mu \mathrm{m}$ SEDs of 2269 likely stellar members that exhibit no excess emission from circumstellar dust disks at $4.5 \mu \mathrm{m}$ or shorter wavelengths. A subset of $\sim 100$ intermediate-mass stars in the lightly obscured Trumpler 14 and 16 clusters have available spectroscopic $T_{\text {eff }}$, measured from the Gaia-ESO survey. We correctly identify the stellar temperature in $85 \%$ of cases, and the aggregate pHRD for all sources returns the same peak in the stellar age distribution as obtained using the spectroscopic $T_{\text {eff }}$. The SED model parameter distributions of stellar mass and evolutionary age reveal significant variation in the duration of star formation among four large-scale stellar overdensities within the CNC and a large distributed stellar population. Star formation began $\sim 10 \mathrm{Myr}$ ago and continues to the present day, with the star formation rate peaking $\lesssim 3 \mathrm{Myr}$ ago when the massive Trumpler 14 and 16 clusters formed. We make public the set of 100,000 SED models generated from standard pre-main-sequence evolutionary tracks and our custom software package for generating pHRDs and mass-age distributions from the SED fitting results.
\end{abstract}

Key words: methods: data analysis - open clusters and associations: individual (Carina Nebula) - stars: formation stars: pre-main-sequence - X-rays: stars

\section{Introduction}

The timescale over which giant molecular clouds (GMCs) collapse and produce new stars places fundamental constraints on theories of star formation (McKee \& Ostriker 2007; Krumholz et al. 2012; Burkhart 2018), calibrations of star formation rates (SFRs; Chomiuk \& Povich 2011; Kennicutt \& Evans 2012), and the dynamical evolution of H II regions (Weaver et al. 1977; Koo \& McKee 1992; Arthur et al. 2011; Zamora-Avilés et al. 2019). Massive GMCs produce the O- and early B-type (OB) stars that dominate feedback on the galactic scale, so measuring the duration of star formation in these structures calibrates the tachometers for the engines driving galaxy evolution (Hopkins et al. 2014, 2018; Vogelsberger et al. 2014).

Our knowledge of constituent stellar populations in the most massive Galactic GMCs has been limited by the combination of relatively large heliocentric distances ( $~ 2 \mathrm{kpc}$ ), relatively large angular extent (typically tens of arcmin to $>1^{\circ}$ in diameter), high differential extinction $\left(\Delta A_{V}>10 \mathrm{mag}\right)$, and overwhelming contamination by unassociated field stars in the Galactic plane (Povich et al. 2011b, 2017). Large archival data sets from the Chandra X-ray Observatory and Spitzer Space Telescope have finally flung open an observational window to identify and resolve the young stellar populations associated with the most massive Galactic GMCs (Benjamin et al. 2003; Townsley et al. 2011b, 2014, 2018; Feigelson et al. 2013; Povich et al. 2013; Wright et al. 2014).

\footnotetext{
${ }^{4}$ Visitor in Astronomy, California Institute of Technology, Pasadena, CA 91125, USA.
}

To constrain the duration of star formation, intermediatemass $\left(2-8 M_{\odot}\right)$ stars are potentially the most valuable, yet still underutilized, segment of the stellar mass distribution. OB stars are relatively rare and reach the zero-age main sequence (ZAMS) while still embedded in their dusty natal cores (Zinnecker \& Yorke 2007). The main-sequence turnoff for OB stars gives the age of individual massive clusters (e.g., Figer et al. 1999; Fall et al. 2005), but cannot probe timescales shorter than the $\sim 3-20$ Myr MS lifetimes of the most massive stars formed in a given cluster and breaks down if massive and low-mass stars are not coeval (Massey \& Hunter 1998). Isochronal ages for low-mass, pre-main-sequence (pre-MS) stars are frequently employed, but these stars evolve relatively slowly along the fully convective Hayashi tracks, requiring very precise placement on the Hertzsprung-Russell (HR) diagram to establish precise ages. Distant GMCs produce additional challenges, as low-mass $\mathrm{T}$ Tauri stars are faint and difficult to detect, and their photospheres are frequently veiled by circumstellar dust in a disk or contaminated by accretion signatures such as strong emission lines (Soderblom et al. 2014). By contrast, intermediate-mass, pre-MS stars (IMPS ${ }^{5}$ ) evolve more rapidly than low-mass T Tauri stars. Some fraction

\footnotetext{
This class of stars, the cooler progenitors of Herbig Ae/Be stars, have also been called intermediate-mass T Tauri stars (IMTTS; Calvet et al. 2004) and G-type T Tauri stars (GTTS; Herbst \& Shevchenko 1999). We introduce the new acronym IMPS because it is more physically descriptive of cool, partially or fully convective stars (Palla \& Stahler 1991). We hope to avoid further overworking the $\mathrm{T}$ Tauri moniker with additional modifiers, considering that two members of the eponymous triple system, T Tauri Sa and T Tauri N, each have masses of $\sim 2 M_{\odot}$ (Köhler et al. 2016), so they are themselves IMPS.
} 
of IMPS shed their circumstellar disks in 1-2 Myr (Hillenbrand et al. 1992; Hernández et al. 2007; Povich et al. 2016) to reveal cool, convective photospheres before transitioning to the blue ZAMS in <10 Myr (Bernasconi \& Maeder 1996; Siess et al. 2000; Haemmerlé et al. 2019). These stars have active dynamos powering magnetocoronal X-ray emission (Gregory et al. 2012, 2016), so they are identifiable as Chandra point sources even when the lack of an inner dust disk means there is no measurable mid-infrared (MIR) excess emission above the stellar photosphere. Although they may still possess debris disks, we will henceforth use the shorthand "diskless" to refer to X-ray-selected young stars with MIR colors consistent with bare photospheres (for $\lambda \leqslant 4.5 \mu \mathrm{m}$ ).

The wide-field, homogeneous X-ray and infrared data sets produced for the Chandra Carina Complex Project (CCCP; Townsley et al. 2011b) provide an ideal test case for our new methodology. The Carina Nebula Complex (CNC) contains a very large population of $\gtrsim 5 \times 10^{4}$ stars arranged hierarchically among three major, massive clusters (Trumpler 14, 15, and 16) and two dozen smaller clusters (including Collinder 228 and 232, Bochum 10 and 11, and the Treasure Chest), and as many as half of the young stars comprise a distributed population (Feigelson et al. 2011; Kuhn et al. 2014). This spatial complexity reflects a complicated star-forming history over at least $10 \mathrm{Myr}$ (DeGioia-Eastwood et al. 2001). The ages of individual subclusters within the CNC span at least several megayears (Feinstein et al. 1980; Carraro 2002; Tapia et al. 2003; Smith et al. 2005; Ascenso et al. 2007; Hur et al. 2012; Getman et al. 2014), and numerous regions of currently active, possibly feedback-driven star formation permeate the remaining molecular cloud material (Smith et al. 2010; Povich et al. 2011a; Roccatagliata et al. 2013). The CNC therefore boasts one of the largest, relatively nearby ( $<3 \mathrm{kpc}$ from the Sun) populations of IMPS, Herbig Ae/Be stars, and intermediate-mass ZAMS stars formed from a single GMC. Our methodology must succeed in reproducing the full range of ages represented by this population while handling the significant differential extinction (negligible $A_{V}$ in certain places, $A_{V}>15 \mathrm{mag}$ in others, with a reddening law that is known to vary with location and with increasing extinction; see Povich et al. 2011b and references therein).

\section{Data, Models, and Source Sample Construction}

\subsection{Source Data Sets}

This pilot study uses previously published CCCP data sets, specifically a large subset of the 4664 Chandra X-ray point sources that were positionally matched to MIR sources from the Spitzer Space Telescope Vela-Carina Survey Point Source Archive (Broos et al. 2011b; Povich et al. 2011a). ${ }^{6}$ The Archive provides broadband photometry data at 3.6, 4.5, 5.8, and $8.0 \mu \mathrm{m}$, plus $J H K_{S}$ near-IR (NIR) photometry from the Two Micron All-Sky Survey (2MASS) Point Source Catalog (Skrutskie et al. 2006), which is well matched to both the $2^{\prime \prime}$ resolution of the Spitzer/IRAC detector (Fazio et al. 2004) and the sensitivity limits of the shallow, Spitzer/ GLIMPSE-style Legacy surveys ([3.6] $\lesssim 15.5 \mathrm{mag}$; Churchwell et al. 2009).

\footnotetext{
6 Vela-Carina survey mosaics and point-source lists were produced using the GLIMPSE pipeline; for details of the processing and data products, go to http://irsa.ipac.caltech.edu/data/SPITZER/GLIMPSE/doc/glimpse1_ dataprod_v2.0.pdf.
}

In the process of constructing the Pan-Carina YSO Catalog of Spitzer/IRAC MIR-excess sources, Povich et al. (2011a) identified 3444 IR counterparts to CCCP X-ray sources that were consistent with normally reddened stellar photospheres, plus 213 sources with "marginal" excess emission in the IRAC [5.8] or [8.0] bands. These 3657 sources form the basis of our source sample, as they were possible X-ray-detected members of the $\mathrm{CNC}$ but did not exhibit significant $4.5 \mu \mathrm{m}$ excess emission above a stellar photosphere.

\subsection{Refinement of CCCP Membership List Using Gaia DR2 Parallaxes}

Broos et al. (2011a) classified $75 \%$ of X-ray point sources in CCCP as probable members of the Carina Nebula young stellar population based on proximity to observed spatial overdensities, X-ray brightness and median energy, and visual/ infrared magnitudes. Kuhn et al. (2019) analyzed proper motion and parallax information from Gaia DR2 (Gaia Collaboration 2018) for 28 young Galactic clusters and associations with available lists of X-ray-selected members, including $\mathrm{CCCP}$, and found that contamination from unrelated field stars was generally $<15 \%$. We perform our own cleaning of the CCCP point-source catalog to remove remaining contaminants using an analysis of the Gaia DR2 parallax distribution, similar to that of Kuhn et al. (2019). We do not consider proper motion information because we do not wish to impose a kinematic membership criterion that might exclude high-velocity stars, for example those ejected from one of the massive clusters but still found within the wide CCCP field of view.

We first identified Gaia DR2 matches to CCCP X-ray sources with 2MASS counterparts (Broos et al. 2011b). The nearest Gaia DR2 source falling within 1"'2 of the 2MASS source was declared matched. We then analyzed the parallaxes of 4053 Gaia DR2 matches with $G>8$ mag and astrometric_excess_noise $<1.0$ mas. The uncertainty on the parallax for each source was adjusted from the DR2 catalog value using the "tentative external calibration" of Lindegren et al. (2018). We then computed the median of the parallax distribution, omitting 417 sources that were $>1 \sigma$ outliers. Our median parallax $\varpi_{0}=0.40 \pm 0.04$ mas gives a distance of $2.50_{-0.23}^{+0.28} \mathrm{kpc}$, in agreement with Kuhn et al. (2019) within the uncertainties, which are dominated by our shared assumption of a 0.04 mas systematic uncertainty in the parallax zero point. This moves the Carina nebula to a larger distance than the $2.3 \mathrm{kpc}$ assumed in the original CCCP studies, but this distance value is still consistent with the lower end of the Gaia DR2 distance estimates. Finally, we flagged 294 CCCP sources with individual parallax measurements $>3 \sigma$ above the median (based on their individual adjusted parallax uncertainties) as foreground stars. Of these, 119 were previously classified as probable members of the Carina complex by Broos et al. (2011a). None of the CCCP sources could be analogously flagged as a background star.

Our parallax-based cleaning of probable foreground stars thus removed $7 \%$ of all IR-bright CCCP sources. Field stars that were undetected by Gaia or detected but with sufficiently high DR2 parallax uncertainties that they could not be confidently removed may persist as residual contaminating sources. 


\subsection{The SED Models}

For this and future papers in this series, we employ a set of $10^{5}$ "naked" pre-MS spectral energy distribution (SED) models that was first described by Povich et al. (2016, hereafter P16). These models are publicly available. ${ }^{7}$ The naked pre-MS model set is similar to the $s-s-i$ model set from Robitaille (2017, hereafter R17). Both of these model sets consist of spherical stellar photospheres only, with no circumstellar dust in a disk, envelope, or ambient medium. The naked pre-MS models use Castelli \& Kurucz (2004) plane-parallel LTE stellar photospheres, the same as the R17 models for $T_{\text {eff }} \geqslant 4000 \mathrm{~K}$. All R17 model sets describe the central sources solely in terms of stellar radius $R_{\text {eff }}$ and temperature $T_{\text {eff }}$, with no reference to a particular set of evolutionary tracks and hence no assignment of stellar mass or age. By contrast, for the naked pre-MS models, we sampled the stellar mass $M_{\star}$, and age $t_{\star}$ values were sampled uniformly in logarithmic space (Robitaille et al. 2006, P16) and then converted to ( $\left.T_{\text {eff }}, R_{\text {eff }}\right)$ space using preMS evolutionary tracks (Bernasconi \& Maeder 1996; Siess et al. 2000). This adds a third derived parameter variable, surface gravity, to the naked pre-MS models that is absent in the $S-s-i$ models, but in practice broadband photometry only constrains the independent variables $R_{\text {eff }}$ and $T_{\text {eff }}$.

The regions of the traditional Hertzsprung-Russell diagram (HRD; $L_{\mathrm{bol}}-T_{\mathrm{eff}}$ space) and of $M_{\star}-t_{\star}$ space sampled by the naked pre-MS models are illustrated in Figure 1. For our goal of constraining age distributions of large stellar populations, both this parameter sampling and the larger size of the naked pre-MS models offer key advantages over the R17 s-s-i models. The uniform sampling in $\log t_{\star}$ maps to the familiar, highly nonuniform distribution on the HRD characterized by the thin, densely populated diagonal line of the ZAMS and the nearly vertical pre-MS overdensity. The density of models is greatly reduced between the ZAMS and the pre-MS, a region that has been called the R-C (radiative-convective) gap (Mayne et al. 2007) because it falls between intermediate-mass mainsequence stars with radiative envelopes and their pre-MS analogs that are still fully or partially convective. The region of the HRD to the lower left of the ZAMS is unphysical and hence unpopulated (Figure 1(a)). These models do not include postmain-sequence evolution, creating an unpopulated region in the upper right of the mass-age parameter space (Figure 1(b)). While much effort continues to be devoted to the further development and refinement of pre-MS evolutionary models (e.g., Somers \& Pinsonneault 2015; Choi et al. 2016; Dotter 2016; Haemmerlé et al. 2019), all stellar populations synthesized from pre-MS evolutionary tracks share these same general qualitative features. The naked pre-MS SED models therefore possess a built-in, physical "prior" probability distribution of where observed young stars are most likely to be placed on the HRD. This comes at the cost of assuming a particular set of pre-MS evolutionary tracks that may not be correct. The evolutionary models used in our SED models are two decades old, but we adopt them because (1) they have been widely used in the literature, particularly in studies of isochronal ages in the $\mathrm{CNC}$ and other Galactic massive starforming regions to which we will directly compare the results from our new method; and (2) most recent innovations in preMS evolutionary models have focused on low-mass stars. We

7 https://doi.org/10.5281/zenodo.2647586
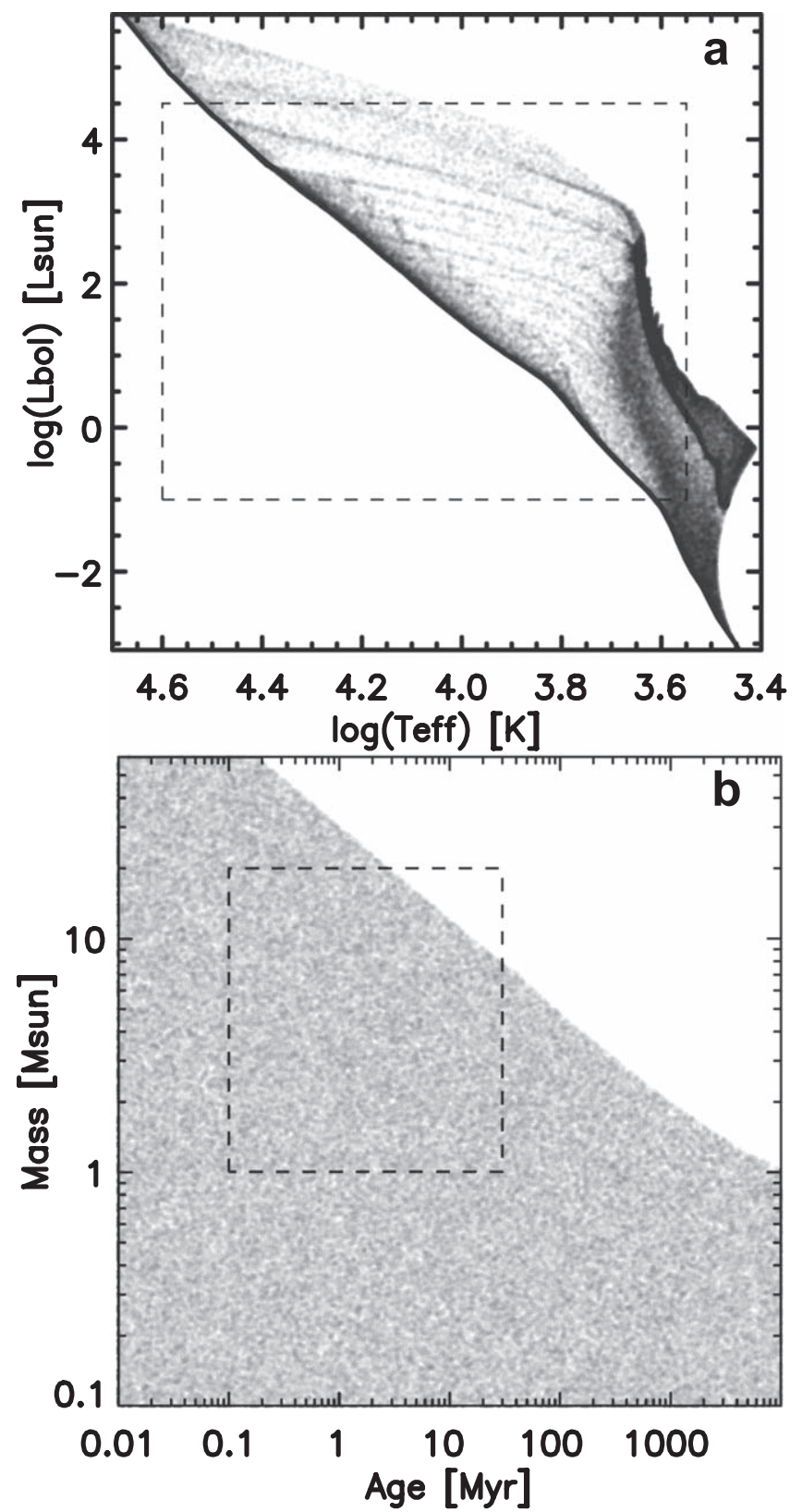

Figure 1. Plots showing parameter spaces sampled by the $10^{5}$ naked stellar models. Each model is plotted as a single gray dot, with transparency such that areas with high density of overlapping models turn darker gray to black. (a) Bolometric luminosity versus effective temperature, replicating a traditional HR diagram. The striped structure visible in the model density is an artifact of the interpolation between the discrete set of pre-PM evolutionary tracks. (b) Stellar mass versus evolutionary age, the fundamental parameter plane used to construct the grid. Dashed boxes on both panels show the boundaries of the parameter ranges of interest for the diskless, IR-bright sources in this study.

discuss how the choice of different evolutionary models could impact our results in Section 5.2.

Figure 2 illustrates the time evolution of the model SEDs for a 1,2 , and $3 M_{\odot}$ star over the first $10 \mathrm{Myr}$. The $1 M_{\odot}$ model star shows no measurable change in SED shape over this timescale, typical of low-mass pre-MS stars that slowly descend the Hayashi tracks at near-constant $T_{\text {eff. }}$ The intermediate-mass model SEDs, by contrast, exhibit a rapid transition from cool, pre-MS stars to hot, A- or B-type ZAMS stars (between 3 and $5 \mathrm{Myr}$ for the $2 M_{\odot}$ evolutionary track and between 1 and 

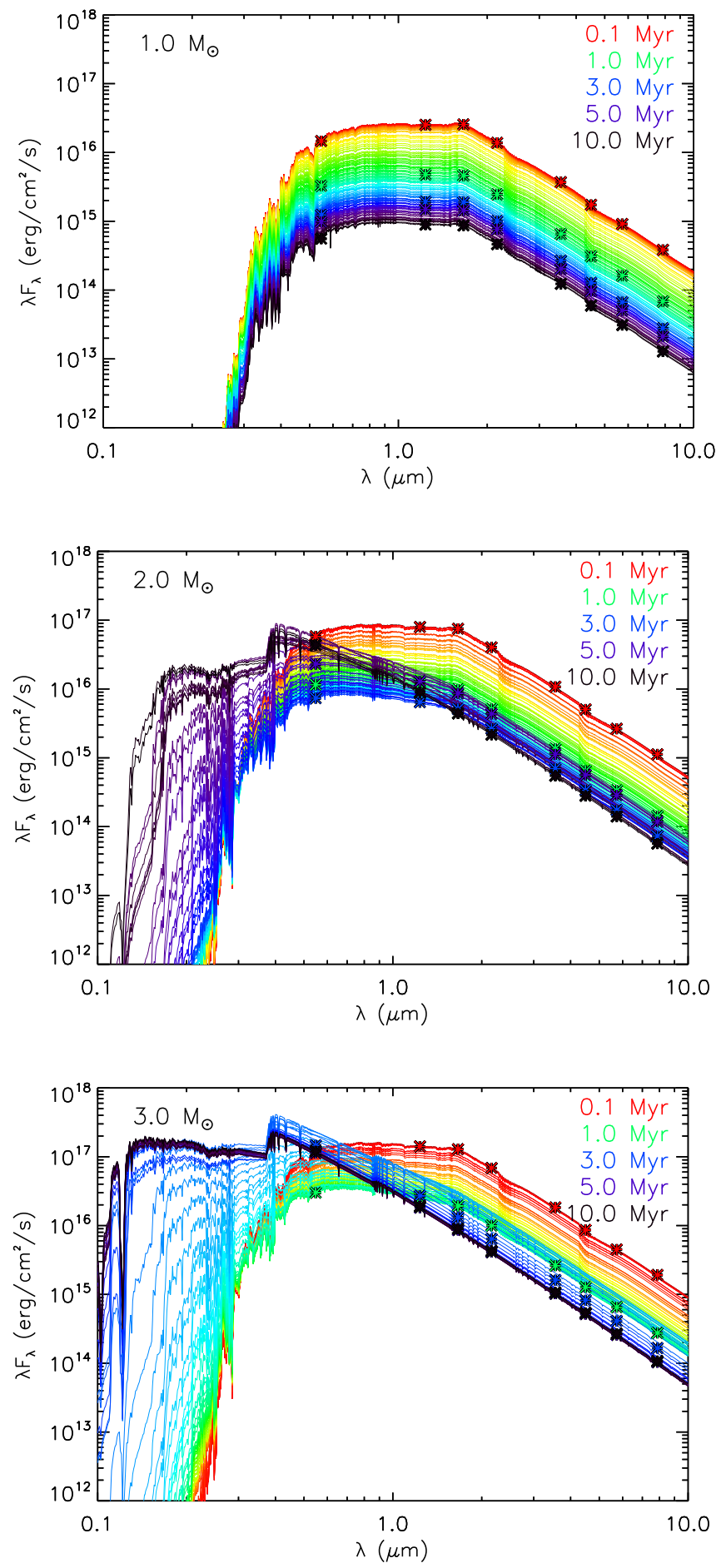

Figure 2. Time evolution of the SED models for the first $10 \mathrm{Myr}$ along the 1, 2, and $3 M_{\odot}$ Siess et al. (2000) tracks. The 100 spectra plotted in each panel are Castelli \& Kurucz (2004) atmospheres, with stellar temperatures and radii sampled at logarithmic age intervals and color coded from red (youngest) through dark violet to black (oldest). Model fluxes convolved with the $V J H K_{s}$ and IRAC bandpasses are overplotted as color-coded asterisks for each isochronal age indicated in the plot legends.

$3 \mathrm{Myr}$ for $3 M_{\odot}$ ). This change in SED shape is measurable using broadband IR photometry. Specifically, $J H K_{S}$ photometry can distinguish between the cool pre-MS and hot ZAMS SEDs, constraining $T_{\text {eff }}$, while Spitzer/IRAC photometry at $\geqslant 3.6 \mu \mathrm{m}$ places strong constraints on the Rayleigh-Jeans tail of the SED and hence $R_{\text {eff }}$ (or $L_{\mathrm{bol}}$ ) for a specified $T_{\text {eff }}$.

\subsection{Fitting Models to the Data}

We fit the SED models to available broadband photometry data using the publicly available Python port (R17) ${ }^{8}$ of the Robitaille et al. (2007) SED fitting tool. All of these CCCPmatched IR sources had been previously fit with different SED models and were found to be consistent with stellar photospheres by Povich et al. (2011a), which imposed the requirement that all sources were detected in at least four of the seven combined 2MASS and IRAC photometry bands, including both the IRAC [3.6] and [4.5] bands. The majority of sources were detected in all five photometry bands from 1 to $4.5 \mu \mathrm{m}$. Because the SED declines toward longer IR wavelengths for these diskless sources and the Carina Nebula itself produces a very bright nebular IR background, detection in the IRAC [5.8] and [8.0] bands was less frequent. For the $\sim 10 \%$ of sources with "marginal" excess emission detected at [5.8] or [8.0], these bands were not used for SED fitting.

The extinction curve of Indebetouw et al. (2005) was applied to the SED models as part of the fitting process, which introduced a third independent parameter variable, $A_{V}$, to our fitting results. Using the standard $J-H$ versus $H-K_{S}$ colorcolor diagram, we determined the maximum extinction to our IR-bright CCCP sources to be $A_{V}=15$ mag and imposed this as a hard upper limit for reddened SED models. We also constrained the scale parameter $R_{\text {eff }} / d$ of the model SEDs by restricting the distance to $2.3 \mathrm{kpc} \leqslant d \leqslant 2.8 \mathrm{kpc}$.

Following our well-established practice, we use the (unreduced) $\chi^{2}$ goodness-of-fit parameter normalized to the number of photometry data points used in the fit $\left(N_{\text {data }}\right)$ to identify sources that can be successfully modeled with our chosen model set (e.g., Povich et al. 2011a, 2013; Robitaille 2017). Because the naked SED models are new, we experimented with different values and found well-fit sources to be those for which the single best-fit model satisfied $\chi_{0}^{2} / N_{\text {data }} \leqslant 1$. We explain our rationale for this choice of cutoff value in Section 3.1.

\subsection{Likelihood-based Weighting of SED Model Fits}

We define the set $i$ of well-fit SED models to each source using

$$
\frac{\chi_{i}^{2}-\chi_{0}^{2}}{N_{\text {data }}} \leqslant 1 .
$$

This is a more strict cutoff than was used by P16, but still typically generates sets of hundreds or even thousands of wellfit models for a given source.

Interstellar dust makes the photometric colors of reddened, hotter stars nearly indistinguishable from those of unreddened, cooler stars, an unfortunate property of nature that greatly complicates observational stellar astronomy. This manifests itself in our SED modeling as a strong degeneracy between $T_{\text {eff }}$ and $A_{V}$. To ameliorate the impact of this degeneracy on our results, we leverage external information about each source in our sample to weight the relative likelihood of each model in the well-fit set. ${ }^{9}$ As described in Appendix B of P16, the relative probability of each individual model $i$ in the set of

\footnotetext{
8 http://doi.org/10.5281/zenodo.235786

9 The software routines and step-by-step procedures used to carry out our advanced analysis of the SED fitting results from this point onward are publicly available as an IDL library at https://doi.org/10.5281/zenodo.3234101. We plan to produce a python port in the future.
} 
well-fit models is given by

$$
P_{i}\left(\tau_{d}, \tau_{X}\right)=P_{n} W_{i}\left(\chi^{2}\right) W_{i}\left(\tau_{d}\right) W_{i}\left(\tau_{X}\right)
$$

(their Equation (5)), in which $P_{n}$ is a normalization constant ensuring that $\sum P_{i}=1$. We compute the first two of the following weighting terms using the functions defined by P16. The likelihood of each model based on goodness of fit is $W_{i}\left(\chi^{2}\right)$ (Equation (4) of P16). The likelihood that a star of a particular mass and age is diskless is $W_{i}\left(\tau_{d}\right)$, computed for each $\left(M_{\star}, t_{\star}\right)$ parameter combination using Equations (6) and (7) of P16. This weighting function disfavors very young, naked SED models for which we would expect the MIR SED to include emission from a circumstellar dust disk or protostellar envelope. The $W_{i}\left(\tau_{d}\right)$ weighting term is only appropriate for sources for which we can be confident that the stellar photosphere dominates the detected MIR emission at $4.5 \mu \mathrm{m}$.

The final weighting term, $W_{i}\left(\tau_{X}\right)$, is the likelihood that the star is X-ray detected based on the model $\left(M_{\star}, t_{\star}\right)$ parameter combination. This term attempts to quantify our knowledge that bright, coronal X-ray emission is a strong indicator of youth. P16 cautioned that their weighting function was strictly appropriate only for low-mass $\mathrm{T}$ Tauri stars for which coronal $\mathrm{X}$-ray emission has been well characterized. However, Gregory et al. (2016) reported coronal X-ray emission for pre-MS stars of all masses in a sample that included stars up to $3 M_{\odot}$, the progenitors of main-sequence A-type stars. These authors also demonstrated that the X-ray luminosity decreases more rapidly with age in more massive pre-MS stars, following the time elapsed since the development of a radiative core in partially convective stars. Using the $L_{X}(t)$ functional relationships for various mass ranges provided by Gregory et al. (2016), we have revised the P16 weighting function based on X-ray detection as follows:

$$
W_{i}\left(\tau_{X}\right)=\left\{\begin{array}{cl}
\left(\frac{t_{\star, i}}{\tau_{X}}\right)^{-\beta_{X}}, & t_{\star, i}>\tau_{X} \\
1, & t_{\star, i} \leqslant \tau_{X} .
\end{array}\right.
$$

We have introduced two new mass-dependent parameters, $\tau_{X}$ and $\beta_{X}$. The critical age at which a radiative core first develops is

$$
\frac{\tau_{X}\left(M_{\star}\right)}{\mathrm{yr}}=\left\{\begin{array}{cc}
10^{6}\left(\frac{1.494}{M_{\star}}\right)^{2.364}, & 1<M_{\star} / M_{\odot} \leqslant 15 \\
2.583 \times 10^{6}, & M_{\star} / M_{\odot} \leqslant 1 .
\end{array}\right.
$$

Once a pre-MS star evolves beyond the critical age, its X-ray emission decays as a power law governed by

$$
\beta_{X}=\left\{\begin{array}{cc}
1.19, & 2<M_{\star} / M_{\odot} \leqslant 15 \\
0.86, & 1.5<M_{\star} / M_{\odot} \leqslant 2 \\
0.75, & M_{\star} / M_{\odot} \leqslant 1.5 .
\end{array}\right.
$$

For low-mass T Tauri stars, $\beta_{X}$ is identical to the value reported by Preibisch \& Feigelson (2005) and adopted by P16. For the IMPS, however, the much more rapid decay in X-ray emission provides significantly stronger $W_{i}\left(\tau_{X}\right)$ constraints on the SED model fitting results.

\subsection{The CCCP IR-bright, Diskless Source Sample}

After selecting only probable members from Broos et al. (2011a), cleaning out remaining foreground contaminants using Gaia DR2 parallaxes, and imposing our goodness-of-fit criterion, our final sample contains 2269 IR-bright, diskless stellar counterparts to CCCP X-ray point sources. The spatial distribution of these probable intermediate-mass members of the CNC young stellar population is shown in Figure 3. The spatial distribution is complex and hierarchical, exhibiting subclustering on multiple size scales (Kuhn et al. 2014; Buckner et al. 2019). Feigelson et al. (2011) divided the clustered CCCP source population into three principal spatial overdensities (regions $\mathrm{A}, \mathrm{B}$, and $\mathrm{C}$, from northwest to southeast) and assigned the remaining sources to a distributed population (region D). To study large-scale variations in the star formation history across the complex, we assign each source in our sample to one of these regions, shown using different-colored symbols enclosed by the lowest density contours in Figure 3. Region A contains the massive $\operatorname{Tr} 15$ and 14 clusters, which are known to have very different ages (e.g., Feinstein et al. 1980; Ascenso et al. 2007), so we bisected this into two subregions, A1 and A2 (separated by the dashed line in Figure 3 ). This yielded $\gtrsim 300$ sources per subregion (1050 sources in the distributed population), sufficiently large samples for robust statistical analysis of the SED fitting results in each.

\section{The Gaia-ESO Spectroscopic Comparison Sample}

Damiani et al. (2017, hereafter D17) analyzed a large set of spectra, obtained as part of the Gaia-ESO survey (Gilmore et al. 2012), for 1085 stars in a relatively small, lightly obscured field containing the massive Trumpler (Tr) 14 and 16 clusters (white box in Figure 3). This stellar sample was selected using the optical photometric catalog of Hur et al. (2012). D17 used a combination of CCCP X-ray detection, radial velocity, and lithium equivalent width $(\mathrm{Li} E W)>$ $150 \mathrm{m \AA}$ to identify 286 likely Carina members with spectral types A or later. D17 published the spectroscopically determined $T_{\text {eff,S }}$ and uncertainties for all of these stars. Of these, 125 were also found within our IR-bright, diskless sample of CCCP sources (white diamonds in Figure 4).

D17 also identified 110 early-type members for which no $T_{\text {eff,S }}$ measurements were possible. Two of these had spectra consistent with obscured O-type stars (previously reported as candidate X-ray-emitting O stars by Povich et al. 2011b), while the rest were likely B-type stars. A large majority of the B-type stars were not detected as CCCP sources, presumably because they are X-ray quiet, and only 14 are found in our sample (orange diamonds in Figure 4). ${ }^{10}$

\subsection{A Grading Scheme for SED Fitting Accuracy}

We utilized this unprecedented, large sample of available spectroscopic classifications for intermediate-mass members of Tr 14 and 16 to benchmark the performance of our SED fitting methodology. For these tests, we included all sources fit with $\chi_{0}^{2} / N_{\text {data }} \leqslant 4$ (expanding our comparison sample to 154 latetype and 19 early-type stars) and performed a second fitting run

\footnotetext{
${ }^{10}$ Because D17 did not tabulate CCCP associations for the small minority of their early-type stars that had them, we instead cross-matched their list to our sources using the 2MASS source IDs.
} 


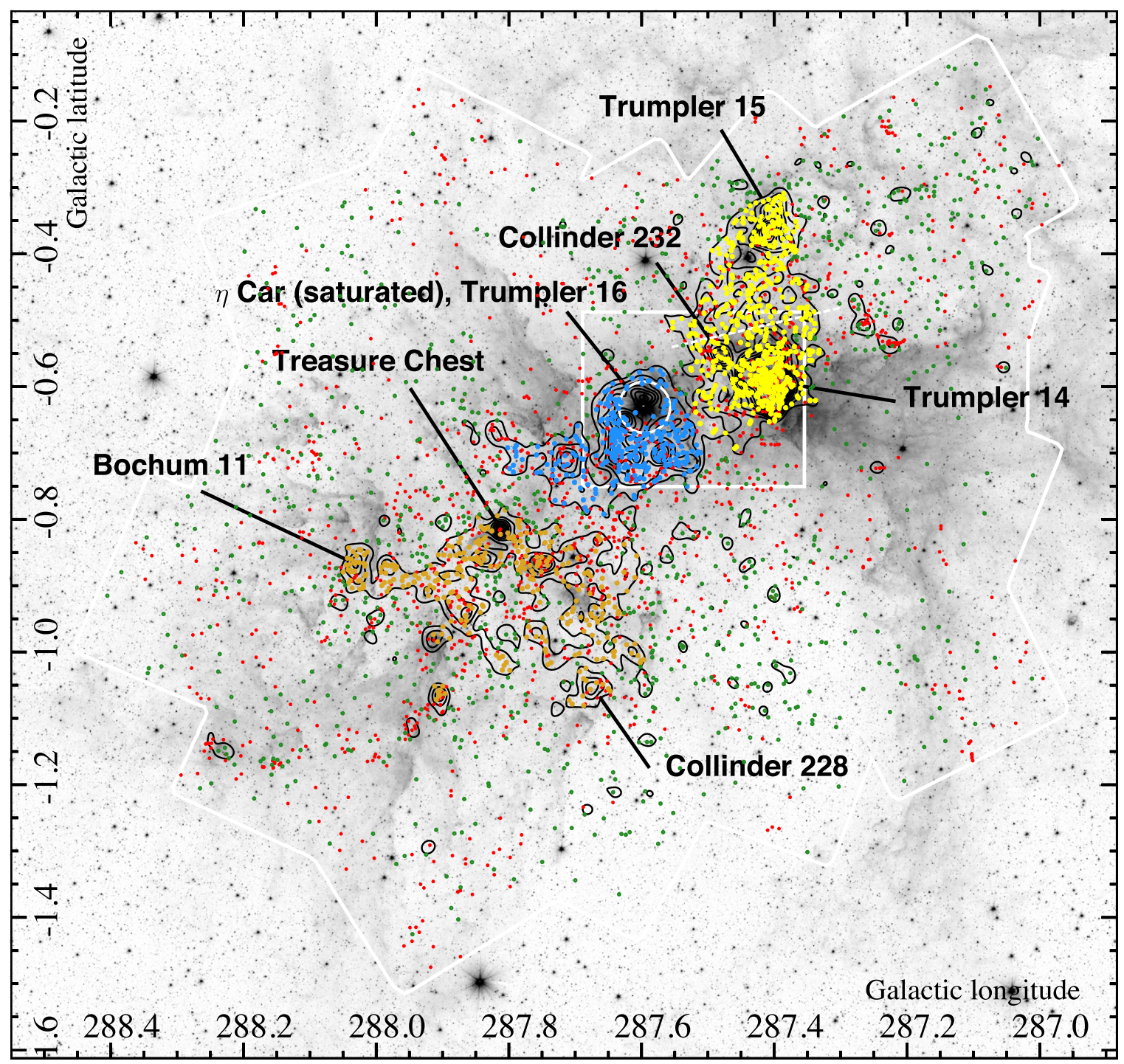

Figure 3. Spitzer/IRAC $3.6 \mu \mathrm{m}$ mosaic image (inverted logarithmic gray scale) with small circles overplotted to mark locations of the intermediate-mass stellar population classified via IR SED fitting. Black contours trace the density of X-ray-identified probable members and reveal three principal subregions with large-area spatial overdensities (Feigelson et al. 2011). The 2269 diskless sources are color coded according to their subregion associations: yellow $=$ Region A (including Tr 14 and 15 and Coll 232; we further separate these sources into regions A1 and A2 above and below the dashed white line); blue = Region B (including Tr 16); orange $=$ Region C (the South Pillars, including Bo 11, Coll 228, and the Treasure Chest); and green = Region D (the distributed population). Disk-bearing young stellar objects (YSOs) from the Pan-Carina YSO Catalog (Povich et al. 2011a) are overplotted as 1432 small red circles; these IR-excess sources are not used in our analysis. IR point-source sensitivity is compromised within a $\sim 1^{\prime}$ radius of the strongly saturated source $\eta$ Car (white circle). The white box encloses the zoomed-in region shown in Figure 4, while the outer white outline marks the boundaries of the CCCP X-ray survey area (Townsley et al. 2011b).

in which we incorporated $V$-band photometry from Hur et al. (2012).

We plotted the $T_{\text {eff }}$ model parameter distributions for each source in the Gaia-ESO comparison sample and compared them to the spectroscopically measured $T_{\text {eff, }}$ values reported by D17. The underlying probability distributions are typically non-Gaussian. Bimodal parameter distributions are not uncommon, reflecting the degeneracy between a hot photosphere with higher reddening or a cooler star with less reddening. We defined a set of accuracy grades from A (excellent agreement between SED fitting and spectroscopy) to F (complete failure to agree). Definitions for the accuracy grades (ABCDF) are given in Table 1. We consider grades ABC to mean the SED modeling successfully reproduced the true $T_{\text {eff,S }}$ of the star, with an accuracy of $\leqslant 10 \%$ for A grades, $\leqslant 30 \%$ for BC grades, and decreasing precision from $\mathrm{A}$ ( $\leqslant 5 \%$ relative uncertainty) to $\mathrm{C}$ grades ( $>10 \%$ relative uncertainty). DF grades, by contrast, reveal strong or irreconcilable disagreement between the SED models and spectroscopy. D17 could not report spectroscopic $T_{\text {eff,S }}$ for early-type stars, but in all cases our SED models also indicated B-type stars, so we consider them the equivalent of $\mathrm{AB}$ grades.

These $T_{\text {eff }}$ accuracy grades guided us to refine our goodnessof-fit criteria (Section 2.4). Among the 154 late-type stars in our expanded spectroscopic comparison sample, those with best-fit $1<\chi_{0}^{2} / N_{\text {data }} \leqslant 4$ represented only $20 \%$ of the sample, indicating a steep decline in fit quality. These relatively poorly fit sources were dominated by DF grades. We therefore adopted $\chi_{0}^{2} \leqslant N_{\text {data }}$ as the robust cutoff for reliable SED fits. This cutoff produced a final Gaia-ESO comparison sample of 139 stars. The secondary SED fitting run, which included $V$-band photometry, reduced the number of well-fit sources by $10 \%$.

The distribution of accuracy grades for the IR-only and $V$ + IR SED fitting runs are tabulated in the $N_{\text {IR }}$ and $N_{\mathrm{V}+\text { IR }}$ 


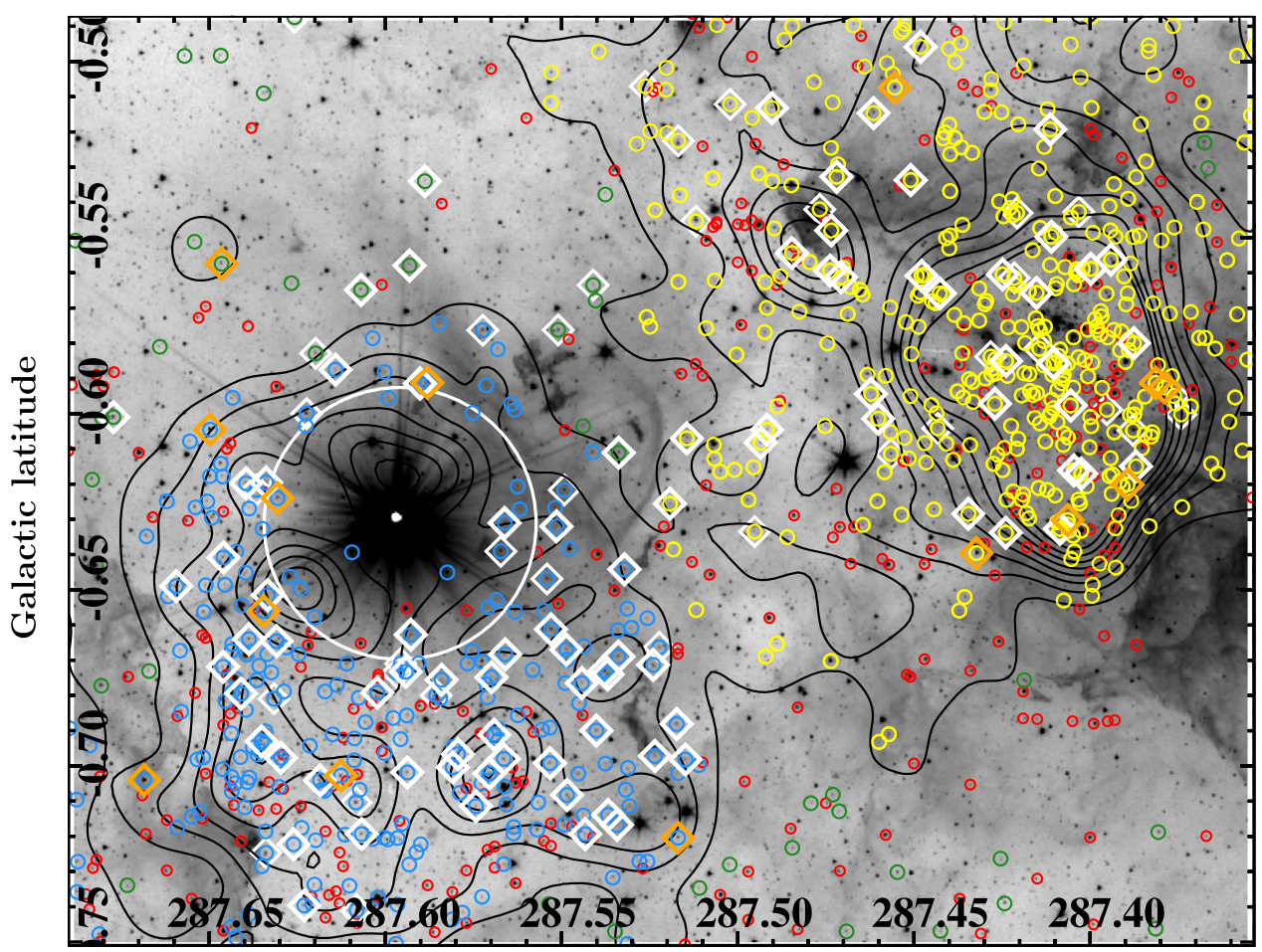

Galactic longitude

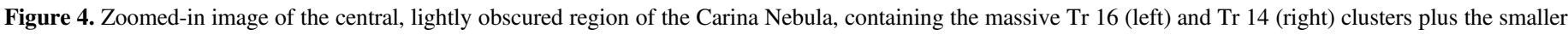

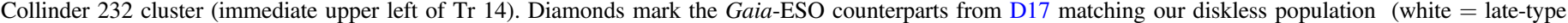
stars and orange $=$ B-type stars). Other overlays are the same as in Figure 3 .

columns, respectively, of Table 1 . In the IR-only fitting of the full Gaia-ESO comparison sample, 97 sources (70\%) received $\mathrm{ABC}$ accuracy grades (this tally includes the 14 early-type stars). Among the well-fit sources in the $V+$ IR fitting run, 92 (74\%) received $\mathrm{ABC}$ grades. This insignificant gain in the accuracy of the SED fitting in reproducing $T_{\text {eff }}$ was produced by the omission of a small number of DF sources that were not well fit when $V$-band photometry was included. The inclusion of visual photometry does tend to more tightly constrain the fitting results, as evidenced by the dozen sources that were promoted from $\mathrm{C}$ to $\mathrm{B}$ grades.

Including $V$-band photometry makes the SED fitting far more sensitive to the shape of the reddening law than is the case with IR photometry alone. The reddening law is known to vary with location in the $\mathrm{CNC}$, especially in the more obscured regions (e.g., Povich et al. 2011b) that occupy a large area on the sky outside of the lightly reddened window studied by Hur et al. (2012) and D17 (see Figure 3). Because the reddening law itself is not a free parameter in our models, any decrease in SED fit quality at visual wavelengths at higher extinction values is unlikely to be outweighed by gains in precision over fitting the IR SED alone. We therefore do not attempt to incorporate visual photometry (for example, from Gaia DR2) into our subsequent analysis of the full CCCP IR-bright sample.

We chose six example sources to represent each of the five SED accuracy grades plus one early-type star. We plot their SED fits in Figure 5 and present their $T_{\text {eff }}$ distributions in Figure 6. The D17 $T_{\text {eff,S }}$ values are overplotted as dashed green lines for comparison to the $P_{i}\left(\tau_{d}, \tau_{X}\right)$-weighted model parameters, illustrating the basis for our accuracy grade assignments. The SED fitting results weighted only by goodness of fit ( $W_{i}\left(\chi^{2}\right)$; red-diamond curves) fail decisively to predict $T_{\text {eff,S }}$ in all of these examples except the early-type star. This is due to the high density of models at cooler temperatures that are inconsistent with the absence of circumstellar disks and envelopes, demonstrating the critical importance of including the $W_{i}\left(\tau_{d}\right)$ parameter from P16.

\subsection{Discrepancies between SED Fitting and Spectroscopy}

In nearly all cases where we assigned DF accuracy grades (including the two examples shown in Figure 6), the SED fitting results preferred a too-hot $T_{\text {eff }}$, equivalent to AB-type stars on the main sequence, while the spectroscopic $T_{\text {eff,S }}$ indicated FGK stars. The simplest explanation for these discrepancies is that we have assumed an incorrect distance in the SED fitting, while the spectroscopic $T_{\text {eff }}$ measurements are distance-independent. Other potential causes for DF grades include erroneous cross matches between our sample and D17 (assumed spectroscopic $T_{\text {eff }}$ is incorrect), unresolved binaries (or confusion between visual and IR sources in crowded areas), or time variability across the several years separating the epochs in which the various visual and IR data sets were obtained.

In Figure 7 we plot Li EW (top panel) and Gaia parallax (bottom panel) versus $T_{\text {eff,s }}$ for the late-type stars in the GaiaESO comparison sample, color coded by SED model accuracy grade. We find that 13 of 15 stars with $\mathrm{Li} \mathrm{EW}<150 \mathrm{m \AA}$ have DF grades (the other two have $\mathrm{C}$ grades). Stars with such low Li EW would have not been selected for membership by D17 if they lacked CCCP X-ray counterparts, increasing the chances that they could be foreground contaminants. However, the parallaxes of the large majority of stars with DF grades, including those with $\mathrm{Li} \mathrm{EW}<150 \mathrm{m \AA}$, exhibit generally 
Table 1

Summary of $T_{\text {eff }}$ Accuracy Grades among the Gaia-ESO Comparison Sample

\begin{tabular}{|c|c|c|c|}
\hline Grade & $N_{\text {IR }(\%)}$ & $N_{\mathrm{V}+\mathrm{IR}(\%)}$ & Definition \\
\hline A & $23(17 \%)$ & $22(18 \%)$ & Tightly constrained $T_{\text {eff }}$ parameter $\left(\sigma_{T} / \overline{T_{\text {eff }}} \leqslant 0.05\right) ; \overline{T_{\text {eff }}}$ within $10 \%$ of $T_{\text {eff,S. }}$ \\
\hline B & $28(21 \%)$ & $40(32 \%)$ & Well-constrained $T_{\text {eff }}$ parameter $\left(\sigma_{T} / \overline{T_{\text {eff }}} \leqslant 0.1\right) ; \overline{T_{\text {eff }}}$ within $30 \%$ of $T_{\text {eff,S. }}$ \\
\hline $\mathrm{C}$ & $32(24 \%)$ & $19(15 \%)$ & Poorly constrained $T_{\text {eff }}$ parameter $\left(\sigma_{T} / \overline{T_{\text {eff }}}>0.1\right) ; \overline{T_{\text {eff }}}$ within $30 \%$ of $T_{\text {eff,S }}$ \\
\hline $\mathrm{D}$ & $32(24 \%)$ & $25(20 \%)$ & Poorly constrained $T_{\text {eff }}$ parameter $\left(\sigma_{T} / \overline{T_{\text {eff }}}>0.1\right) ; \overline{T_{\text {eff }}}$ deviates $>30 \%$ from $T_{\text {eff,S }}$ \\
\hline $\mathrm{F}$ & $10(7 \%)$ & $8(6 \%)$ & Well-constrained $T_{\text {eff }}$ parameter $\left(\sigma_{T} / \overline{T_{\text {eff }}} \leqslant 0.1\right) ; \overline{T_{\text {eff }}}$ deviates $>30 \%$ from $T_{\text {eff,S }}$ \\
\hline [Early] & $14(10 \%)$ & $11(9 \%)$ & Spectra and SED modeling agree on B-type star (AB grade equivalent). \\
\hline
\end{tabular}

Note. $\overline{T_{\text {eff }}}$ and $\sigma_{T}$ are the $P_{i}\left(\tau_{d}, \tau_{X}\right)$-weighted mean and standard deviation, respectively, of the $T_{\text {eff }}$ parameter distribution for each source.

smaller error bars and less scatter about the CCCP median value compared to the distribution of sources with $\mathrm{ABC}$ grades, strongly suggesting that they are $\mathrm{CNC}$ members, not foreground contaminants.

Stars in the cool comparison sample with $5600 \mathrm{~K}<T_{\text {eff,S }}<$ $8000 \mathrm{~K}$ are dominated by DF grades (Figure 7), but their lower $\mathrm{Li}$ EW is consistent with their earlier spectral types (A6 through G5; Pecaut \& Mamajek 2013), and it is not necessarily indicative of the older ages expected of field contaminants. These stars are also visually brighter than typical of the comparison sample, which may explain their relatively low Gaia parallax uncertainties. They have $\log L_{\mathrm{bol}} / L_{\odot} \gtrsim 1$, placing them in a region of the HR diagram where the density of the naked stellar models is minimal (Figure 1, top panel). The SEDs of stars in this region of parameter space are also well fit by ZAMS models at just slightly higher $A_{V}$. Because the model density near the ZAMS is very high, the distribution of well-fit models becomes incorrectly skewed toward higher $T_{\text {eff }}$ values. We suspect this effect is responsible for the majority of the DF grades in the Gaia-ESO comparison sample, and we discuss its impact on our results in the next section.

\section{Results: Probabilistic HR Diagrams}

Thus far we have examined only the SED fitting results for individual sources. However, we are primarily interested in the aggregate properties of stellar populations. We obtain these by summing the normalized SED model parameter distributions of all constituent sources within the various subsets of interest.

\subsection{Temperature and Age Distributions for the Spectroscopic Comparison Sample}

The aggregate $T_{\text {eff }}$ parameter distribution for the late-type stars in the cleaned spectroscopic comparison sample is provided in Figure 8. This plot shows that our weighted SED fitting parameters (black histogram) successfully reproduce the peak in the spectroscopic temperature distribution near $\log T_{\text {eff }} /[\mathrm{K}]=3.7$. The $T_{\text {eff }}$ distribution weighted only by $\chi^{2}$ (red histogram) is unsuccessful, producing a large peak at cooler temperatures that would indicate disk-bearing stars, and a smaller peak at the correct temperature. Irrespective of the weighting function used, SED fitting produces a spurious hump in the distribution with $\log T_{\text {eff }} /[\mathrm{K}]>4$, corresponding to the $30 \%$ of sources with DF accuracy grades.

We also construct joint probability distributions of $T_{\text {eff }}$ and $L_{\text {bol }}$ and plot them directly on the traditional HR diagram as twodimensional histograms. Summing the probability distributions for all of the individual stars in the spectroscopic comparison sample, we obtain the probabilistic HR diagram (pHRD; Figure 9).
pHRDs give information similar to a traditional color-magnitude diagram (CMD), and indeed they appear qualitatively similar to the distribution of points on the $V_{0}$ versus $(V-I)_{0} \mathrm{CMD}$ presented by D17 for the entire Gaia-ESO sample (their Figure 13), of which our comparison sample is a subset. The pHRD represents a philosophical shift away from using a single color and magnitude for each individual star to infer the ensemble properties of a larger population. Instead, we synthesize a model for the ensemble population, using a multidimensional set of colors and magnitudes for each constituent star.

In Figure 9 we compare pHRDs produced using two different weighting methods for the SED fitting results: (top panel) our standard, age-based likelihood weighting $\left({ }_{P i}\left(\tau_{d}, \tau_{X}\right)\right.$; Section 2.5) and (bottom panel) a Gaussian weighting factor $W_{i}\left(T_{\text {eff,S }}\right)$ using $T_{\text {eff,S }}$ and its uncertainty reported by D17. The weighting function in the latter case is

$$
P_{i}\left(T_{\mathrm{eff}, \mathrm{S}}\right)=P_{n} W_{i}\left(\chi^{2}\right) W_{i}\left(T_{\mathrm{eff}, \mathrm{S}}\right),
$$

which typically (and unsurprisingly) yields much tighter constraints on all model parameters for individual sources. While both pHRDs place their pre-MS loci between the 1 and $3 \mathrm{Myr}$ isochrones, the shapes of these loci are noticeably different between the two plots. The peak probability in the $P_{i}\left(\tau_{d}, \tau_{X}\right)$-weighted case forms a narrow ridge with $3.65<$ $\log T_{\text {eff }} /[\mathrm{K}]<3.7$, which extends in the $\log L_{\text {bol }}$ direction upward from the $2 M_{\odot}$ evolutionary track to nearly the $3 M_{\odot}$ track (red part of the heat map in the left panel, with $>8$ stars per bin). The pre-MS locus in the ${ }_{P i}\left(T_{\text {eff,S }}\right)$-constrained case, by contrast, extends parallel to the isochrones, and its peak is much sharper, located on the $2 M_{\odot}$ track just above the $3 \mathrm{Myr}$ isochrone (right panel, $\sim 14$ stars per bin). Two other features of the pHRD produced using our likelihood weighting (Figure 9, top panel) are missing from the $T_{\text {eff, } \mathrm{S}}$-constrained pHRD (bottom panel): (1) a residual locus of cool $\left(\log \left(T_{\text {eff }} /\right.\right.$ $[\mathrm{K}])<3.6)$, low-mass $\left(M_{\star}<1 M_{\odot}\right)$ pre-MS stars and (2) the intermediate-mass (3-7 $M_{\odot}$ ) ZAMS. Our standard likelihood procedure tends to be relocated with DF grades relocated from the hot end of the pre-MS $\operatorname{locus}\left(\log T_{\text {eff,S }} /[\mathrm{K}] \approx 3.75\right)$ to the ZAMS (see also Figure 7). Because this displacement generally parallels the isochrones, the age determination of the population is unaffected, but the masses of stars with DF grades are overestimated.

Because pre-MS tracks and isochrones are built into the SED models, we can trivially transform the pHRDs (Figure 9) into probability distributions of $t_{\star}$ and $M_{\star}$ (Figures 10 and 11). Compared to the pHRD (or the traditional HRD), these transformations offer more straightforward visualizations of 


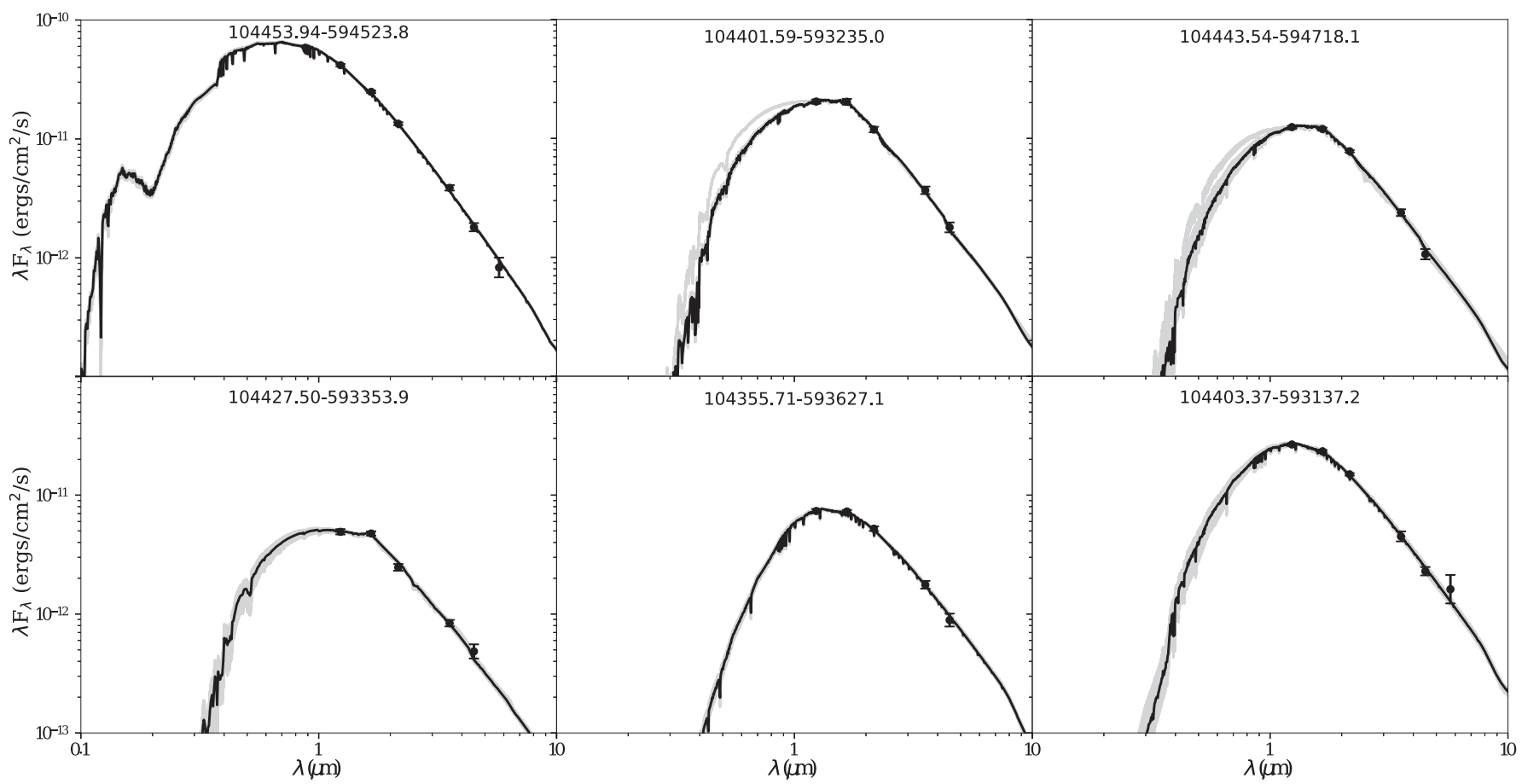

Figure 5. SED fits to the 1-8 $\mu \mathrm{m}$ 2MASS +IRAC IR photometry for six example sources. In each panel, the 200 best-fitting SED models are plotted, with the black curve highlighting the model with the minimum $\chi^{2}$.

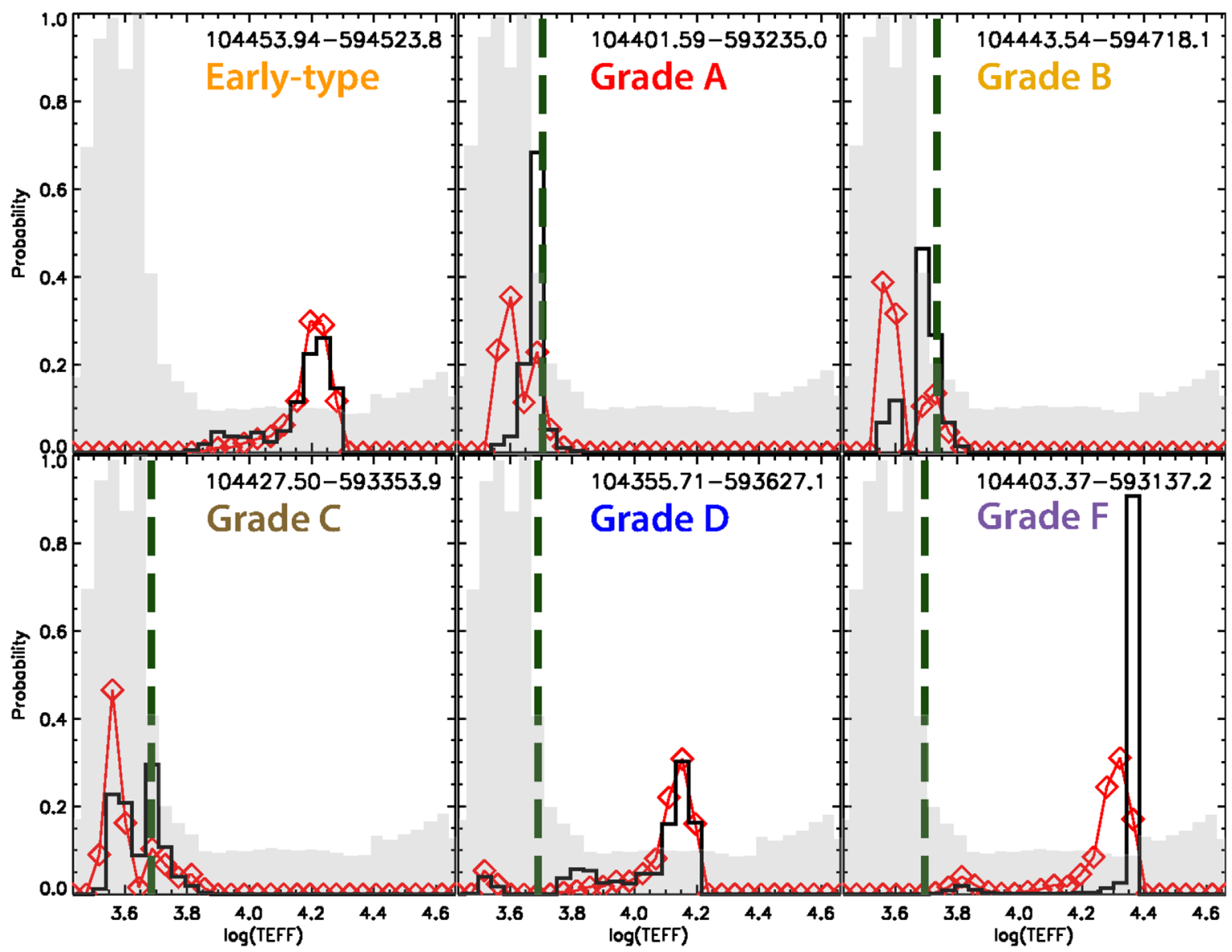

Figure 6. Distribution plots of the $T_{\text {eff }}$ parameter for the six example SED fits shown in Figure 5. Each source exemplifies one of the SED accuracy grades described in Table 1. Three histograms are plotted in each panel: the distribution of all models in the naked pre-MS set (gray, same in all panels); the model fitting results weighted by $W_{i}\left(\chi^{2}\right)$ only (red); and the final model fitting results incorporating all weighting factors in $P_{i}\left(\tau_{d}, \tau_{X}\right)$ (black). The thick, vertical long-dashed green lines mark the spectroscopic $T_{\text {eff }}$ from D17, the typical uncertainties on which are comparable to the size of one histogram bin in these plots. 
the fundamental stellar parameter distributions. To construct these distributions, we bin the well-fit models uniformly in logarithmic intervals, sampling across the full range of each parameter dimension (Figure 1) and applying the $P_{i}\left(\tau_{d}, \tau_{X}\right)$ weighting functions described in Section 2.5 to each source. We experimented with different numbers of bins for each parameter and chose an optimal binning for the composite histograms that provided the smallest bin size for which fluctuations between adjacent bins did not appear dominated by noise. The final binnings chosen for the 2D pHRDs and associated 1D distributions of $M_{\star}$ and $t_{\star}$ were based on the total number of sources included in each distribution.

We create and analyze age and mass distributions (except for the $P_{i}\left(T_{\text {eff,S }}\right)$-constrained models) using a two-step iterative process. In the first iteration, we identify the modal bin where $M_{\star}=M_{C 1}$ in the mass distribution produced with our standard $P_{i}\left(\tau_{d}, \tau_{X}\right)$ likelihood weighting. We include only those models with $M_{\star} \geqslant 1.8 M_{\odot}$ in the $t_{\star}$ distributions (Figure 10). We adopted this cutoff mass to avoid biasing the age distributions toward the youngest pre-MS stars. It also removes the spurious locus of too-cool models with $\log T_{\text {eff }} /[\mathrm{K}]<3.6$ (Figures 7 and 9). The relatively shallow $2 \mathrm{MASS}$ and Vela-Carina surveys are insensitive to ZAMS stars fainter than early $\mathrm{F}$ dwarfs at the distance of the CNC. Younger, low-mass pre-MS stars are larger and cooler, so they may be detected and included in our sample, while older stars of the same mass fall below our IR photometric sensitivity limits. The existence of fainter, low-mass stars can be inferred from the age distributions of intermediate-mass stars.

In the case of a star-forming event that commenced at time $\tau_{\mathrm{SF}}$ (Myr) in the past and proceeded with approximately constant SFR to the present day, logarithmically binned age distributions should exhibit a linearly increasing number of stars per bin as $t_{\star}$ increases from 0 to $\tau_{\mathrm{SF}}$, with a sharp break in the distribution for $t_{\star}>\tau_{\text {SFF }}$. To locate this break point in our age distributions, we identify the modal bin in the $t_{\star}$ histogram (Figure 10). The duration of star formation $\tau_{\mathrm{SF}}$ is the geometric mean of the central values for this modal bin and the adjacent bin in the direction of increasing $t_{\star}$. The uncertainty on $\tau_{\mathrm{SF}}$ is the geometric mean of the widths of these two bins.

Both $t_{\star}$ distributions for the comparison sample give the same value for $t_{\mathrm{SF}}=2.7 \pm 0.8 \mathrm{Myr}$ (Figure 10). While spectroscopy sharpens the age distribution, our likelihood-weighted SED fitting to X-ray-selected, diskless sources accurately measures the duration of star formation from broadband IR photometry alone.

Three model mass distributions for the spectroscopic comparison sample are plotted in Figure 11. The distribution created with our standard age-based weighting function (top panel) follows a Salpeter power law for $M_{\star} \geqslant 2.7 M_{\odot}=M_{C}$, turning over because of photometric incompleteness at lower masses. However, the $P_{i}\left(T_{\text {eff,S }}\right)$-constrained mass distribution, which is both more precise and more accurate, appears strikingly different. The peak of the distribution narrows, shifting to a marginally lower $M_{C}=2.3 M_{\odot}$ and producing a significant deficit of stars with $M_{\star}>3 M_{\odot}$ when compared to the Salpeter slope (the area between the solid line and histogram in the bottom panel). Sample bias introduced by our X-ray selection criteria explains this observed deficit. Intermediate-mass $\left(2-8 M_{\odot}\right)$ stars of types $\mathrm{A}$ and late $\mathrm{B}$ on the MS have no known mechanism for producing detectable X-ray emission and hence occupy an "X-ray desert" between cooler stars with coronal emission (Preibisch et al. 2005) and hotter
OB stars producing X-rays via shocks driven by strong winds (Gagné et al. 2011). But those with X-ray-bright T Tauri companions (e.g., Evans et al. 2011) can be selected for inclusion in our IR-bright, diskless sample.

To better constrain the model mass distribution in the general case where no $T_{\text {eff,S }}$ is available, we iterated the analysis of the SED fitting results, introducing a new Gaussian weighting parameter $W_{i}\left(\tau_{\mathrm{SF}}\right)$. The complete weighting function for this second iteration is hence

$$
P_{i}\left(\tau_{d}, \tau_{X}, \tau_{\mathrm{SF}}\right)=P_{n} W_{i}\left(\chi^{2}\right) W_{i}\left(\tau_{d}\right) W_{i}\left(\tau_{X}\right) W_{i}\left(\tau_{\mathrm{SF}}\right)
$$

To determine the present-day mass function of a stellar population from broadband photometry, it is a common practice to construct a dereddened luminosity function and subsequently employ a mass-luminosity relation appropriate to the age of the population (Offner et al. 2014). Here we have adopted $\tau_{\mathrm{SF}}$ from the first iteration as the best isochrone for converting from $L_{\mathrm{bol}}$ to $M_{\star}$ for our ensemble population, producing the mass distribution plotted in the center panel of Figure 11.

The two most important metrics for the modeled $M_{\star}$ distributions are the location $\left(M_{C}\right)$ and normalization of the scaled Salpeter-type initial mass function (IMF). Scaling a standard IMF model (Kroupa \& Weidner 2003) to the isochronally weighted distribution predicts the same number of intermediate-mass stars as scaling to the spectroscopically constrained distribution (Figure 11, center versus bottom panels) to within $5 \%\left(N_{\mathrm{IM}} \approx 150\right.$ for $M_{\star}>$ $1.8 M_{\odot}$; Table 2). The same IMF scaled to the mass distribution produced using $P_{i}\left(\tau_{d}, \tau_{X}\right)$ weights alone predicts $\sim 30 \%$ fewer stars. Poorer constraints on the mass of individual stars broadens the aggregate mass distribution, mimicking a shallower IMF slope and reducing the peak height near the cutoff mass $M_{C}$ (Figure 11, top). We therefore choose to employ the second iteration to leverage isochronal weighting for $M_{\star}$ distributions for the full CCCP IR-bright, diskless source sample.

We caution that our omission of disk-bearing YSOs and $\mathrm{X}$-ray-quiet stars makes this sample formally incomplete at all masses. These mass distributions should not be used to make measurements that require completeness over some mass interval, for example circumstellar disk fractions or the total size of the CNC stellar population. They are mainly useful for drawing comparisons among the various CCCP subpopulations.

\subsection{Age and Mass Distributions for the CCCP Subregions}

Having validated our methodology on the spectroscopic comparison sample, we applied the same analysis to each of our five CCCP spatial subregions (Figure 3). In Figures 12-16, we present the pHRDs, age, and mass distributions for the sources in each subregion. Each pHRD is annotated with the total number $N$ of stars in the sample, and Siess et al. (2000) isochrones and evolutionary tracks are overlaid (same as in Figure 9). Vertical lines overplotted on the age and mass distributions give the duration of star formation $\tau_{\mathrm{SF}}$ and break mass $M_{C}$, respectively (as in Figures 10 and 11). These and other important quantities describing each subregion are listed in Table 2.

Previous studies have found, based on multiple lines of evidence, that $\operatorname{Tr} 15$ is significantly older than $\operatorname{Tr} 14$ and 16, the other two massive clusters in the CNC (Tapia et al. 2003; Wang et al. 2011). We confirm a significantly earlier onset of star formation in $\operatorname{Tr} 15$ compared to most of the rest of the CNC, with $\tau_{\mathrm{SF}}=6.5 \pm 1.3 \mathrm{Myr}$, in good agreement with the measurements reported by Feinstein et al. (1980) from UBVRI photometry and 

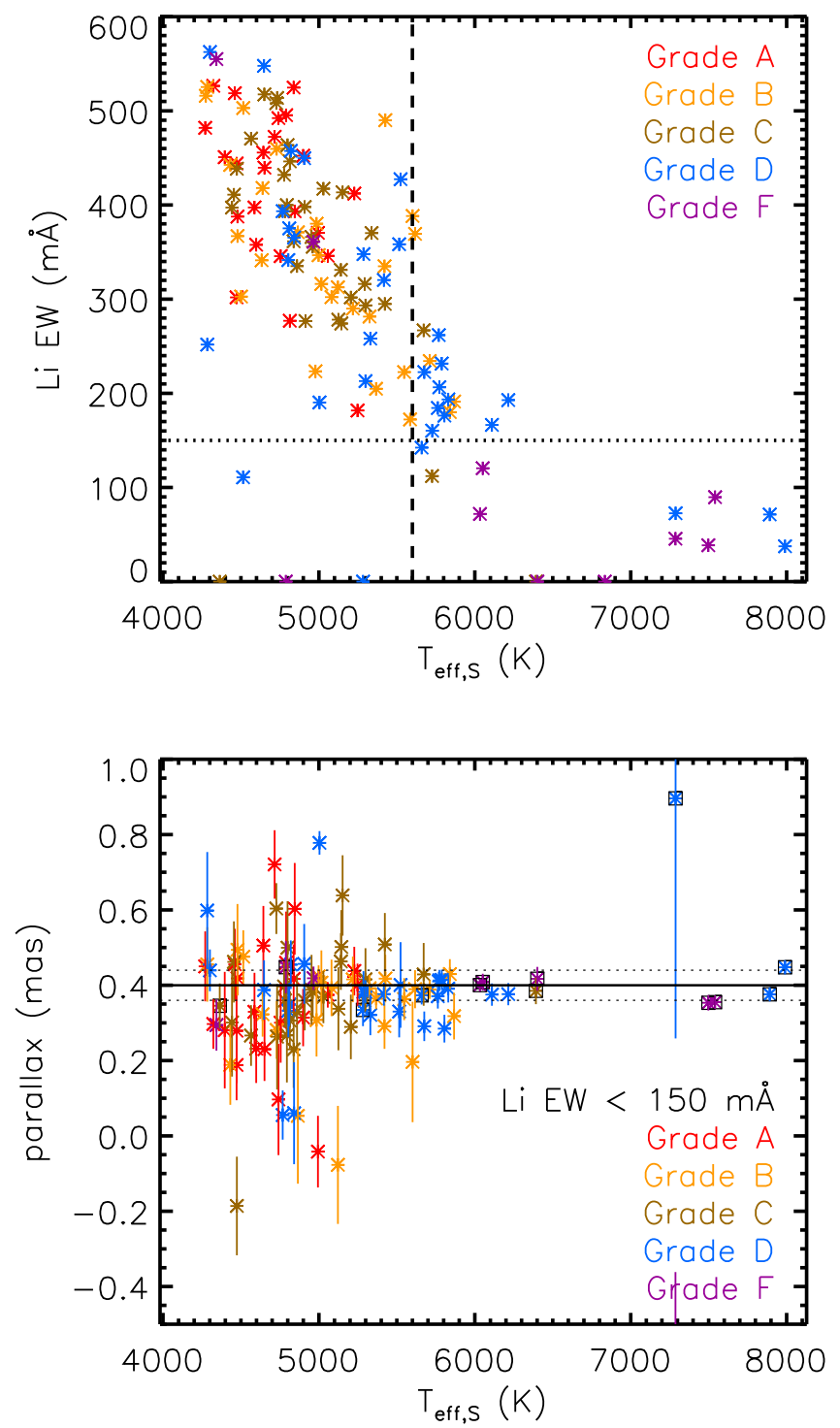

Figure 7. Top: plot of Li equivalent width versus stellar effective temperature for all stars in the Gaia-ESO cool comparison sample. Symbols are color coded by their SED fitting grade. The dotted horizontal line shows the cutoff $\mathrm{Li}$ EW $>150 \mathrm{~mA}$ used by D17 for membership selection, while the vertical dashed line as $T_{\text {eff, }}=5600 \mathrm{~K}$ provides a rough dividing line between regions dominated by DF and ABC SED accuracy grades. Bottom: plot of Gaia DR2 parallax versus stellar effective temperature (12 stars are omitted because they lack parallax measurements, and one low and one high parallax outlier fall outside the bounds of the plotting range). Black boxes mark sources with Li EW $<150 \mathrm{~mA}$. Horizontal solid and dotted lines show the median parallax and uncertainty of $0.40 \pm 0.04$ for our sample of CCCP stellar members (Section 2.2).

spectroscopy of its brightest members. Tr 15 does not contain a large population of IMPS, as most stars with $M_{\star}>2 M_{\odot}$ have already reached the ZAMS (Figure 12). Disk-bearing, bright YSOs are absent from Tr 15 (Povich et al. 2011a).

We find $\tau_{\mathrm{SF}}=2.6 \pm 0.5 \mathrm{Myr}$ for subregions $\mathrm{A} 2$ and $\mathrm{B}$, containing $\operatorname{Tr} 14$ and $\operatorname{Tr} 16$, respectively, each presenting a very luminous pre-MS populated by $2-3 M_{\odot}$ IMPS (Figures 13 and 14). Our results agree broadly with previous studies (e.g., Tapia et al. 2003; Ascenso et al. 2007; Hur et al. 2012). Several studies have reported even younger ages $<2 \mathrm{Myr}$ for the dense core of $\operatorname{Tr} 14$ (Ascenso et al. 2007; Getman et al. 2014; D17), and we do not dispute these results. A very young age is consistent with the large concentration of luminous YSOs (Povich et al. 2011a) and

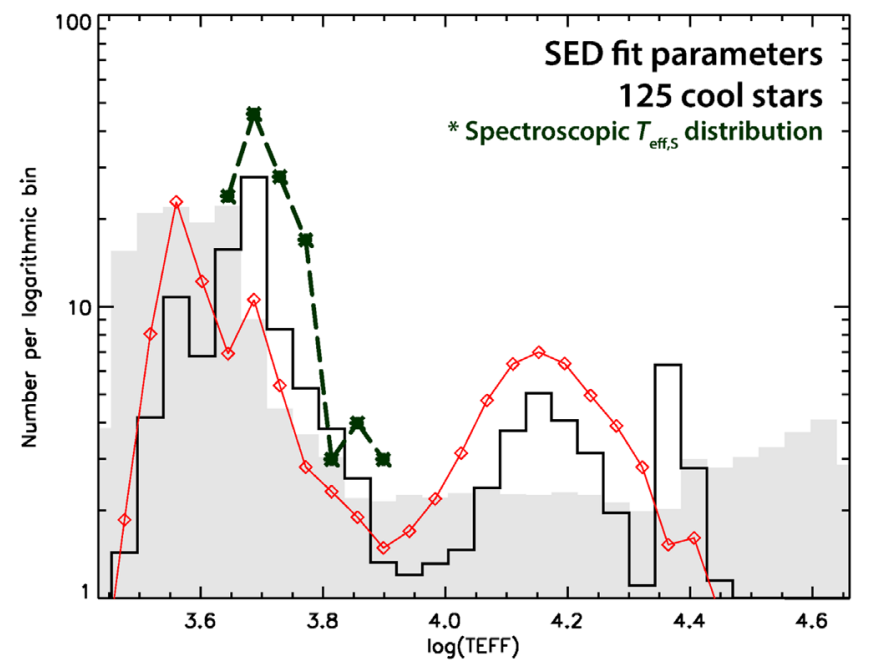

Figure 8. Combined $T_{\text {eff }}$ distributions for all SED fits to the cool spectroscopic comparison sample. Colors and line/histogram styles are the same as in Figure 6.

X-ray-bright IMPS (E. H. Nuñez et al. 2019, in preparation) in $\operatorname{Tr} 14$. Because we selected diskless stars, our sample was biased (by design) toward more-evolved objects. The core of $\operatorname{Tr} 14$ is confused in the 2MASS and Spitzer/IRAC images, so our present analysis can only probe the outskirts of the cluster. Our $M_{\star}$ distributions for both subregions A2 and B break from the Salpeter slope below a relatively high $M_{C}=3.0 M_{\odot}$, indicating severe photometric incompleteness caused by the combination of crowding ( $\operatorname{Tr} 14)$, proximity to the very bright IR source $\eta$ Car (Tr 16), and bright, diffuse background IR nebulosity (both).

Most of the ongoing star formation traced by Spitzer YSOs in the CNC occurs in the South Pillars region, where feedback from massive stars continues to sculpt the remaining GMCs (Smith et al. 2010; Povich et al. 2011a; Roccatagliata et al. 2013). The $\mathrm{X}$-ray-bright, diskless population, however, reveals that the evident embedded population intermingles with a significantly more evolved population $\left(\tau_{\mathrm{SF}}=4.1 \pm 0.8 \mathrm{Myr}\right.$; Figure 15$)$ that predates the $\operatorname{Tr} 16$ and 14 clusters. Ages varying from 1.1 to 4.3 Myr among the various subclusters in the South Pillars were reported by Getman et al. (2014). Individual subclusters display varying disk fractions as well, indicated qualitatively by the varying counts of YSOs versus diskless sources in each one (red versus goldenrod dots in Figure 3). Bochum 11 has few associated YSOs, Collinder 228 has relatively more, and an unnamed, obscured cluster to the immediate southwest of the Treasure Chest hosts many YSOs. The Treasure Chest itself is perhaps the youngest embedded cluster in the CNC (Smith et al. 2005), but its density and high MIR nebulosity precluded detection of its YSO population using Spitzer.

Half of the CCCP stellar members lie outside of the three main spatial overdensities in a distributed population (Region D; Feigelson et al. 2011). Not all of this population is truly distributed; some localized overdensities have been grouped into small subclusters (Kuhn et al. 2014; Buckner et al. 2019). YSOs mixed within the distributed population and its most obvious smaller subclusters (Figure 3) reveal that region D traces a wide range of ages, likely spanning the full star-forming history of the CNC. This age range is reflected in the relatively broad peak of the $t_{\star}$ distribution in Figure 16. Compared to the other subregions, we have decreased the histogram bin size and adjusted the break point, yielding $\tau_{\mathrm{SF}}=7.2 \pm 2.3 \mathrm{Myr}$ for Region $\mathrm{D}$. We find that 


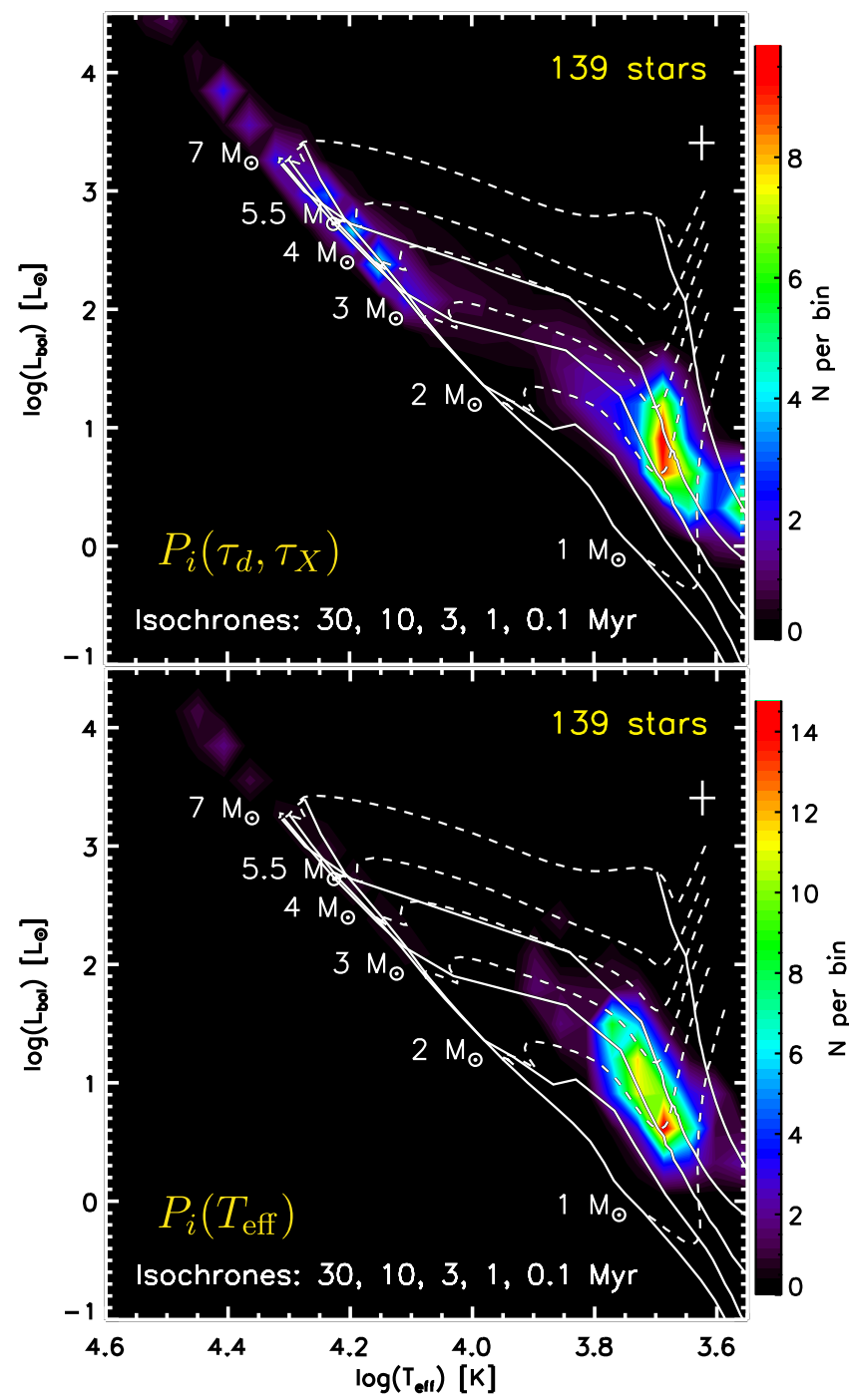

Figure 9. Probabilistic HR diagrams for all SED fits to the spectroscopic comparison sample. Siess et al. (2000) isochrones and evolutionary tracks are plotted as solid and dashed curves, respectively, for the indicated stellar masses and ages. The white crosses in the upper right give the $2 \mathrm{D}$ histogram bin sizes. Top: results from fitting IR-only SEDs using our standard $P_{i}\left(\tau_{d}, \tau_{X}\right)$ weighting function. Bottom: same SED fits, but using the $P_{i}\left(T_{\text {eff }}\right)$ weighting function to constrain the fitting results for the $125 \mathrm{cool}$ stars using spectroscopic data from D17 (the 14 earlytype stars without $T_{\text {eff }}$ measurements are weighted only by $\left.W_{i}\left(\chi^{2}\right)\right)$.

the duration of star formation in the $\mathrm{CNC}$ distributed population, while dominated by the most evolved stars in our CCCP IR-bright, diskless sample, is comparable to the age of the most evolved massive cluster, $\operatorname{Tr} 15$.

\section{Discussion}

\subsection{The Complicated Star Formation History of the CNC}

Our $\tau_{\mathrm{SF}}$ results (Table 2) reveal that star formation began at different times at different locations within the $\mathrm{CNC}$, producing a stellar population with a hierarchical, multiclustered structure. Various locations within the CNC continue to host ongoing star formation, as evidenced by protostellar populations (Povich et al. 2011a; Roccatagliata et al. 2013). Our age distributions (with the possible exception of Region A1) would be populated to the lowest measurable $t_{\star}$ if YSOs with IR excess emission were included.

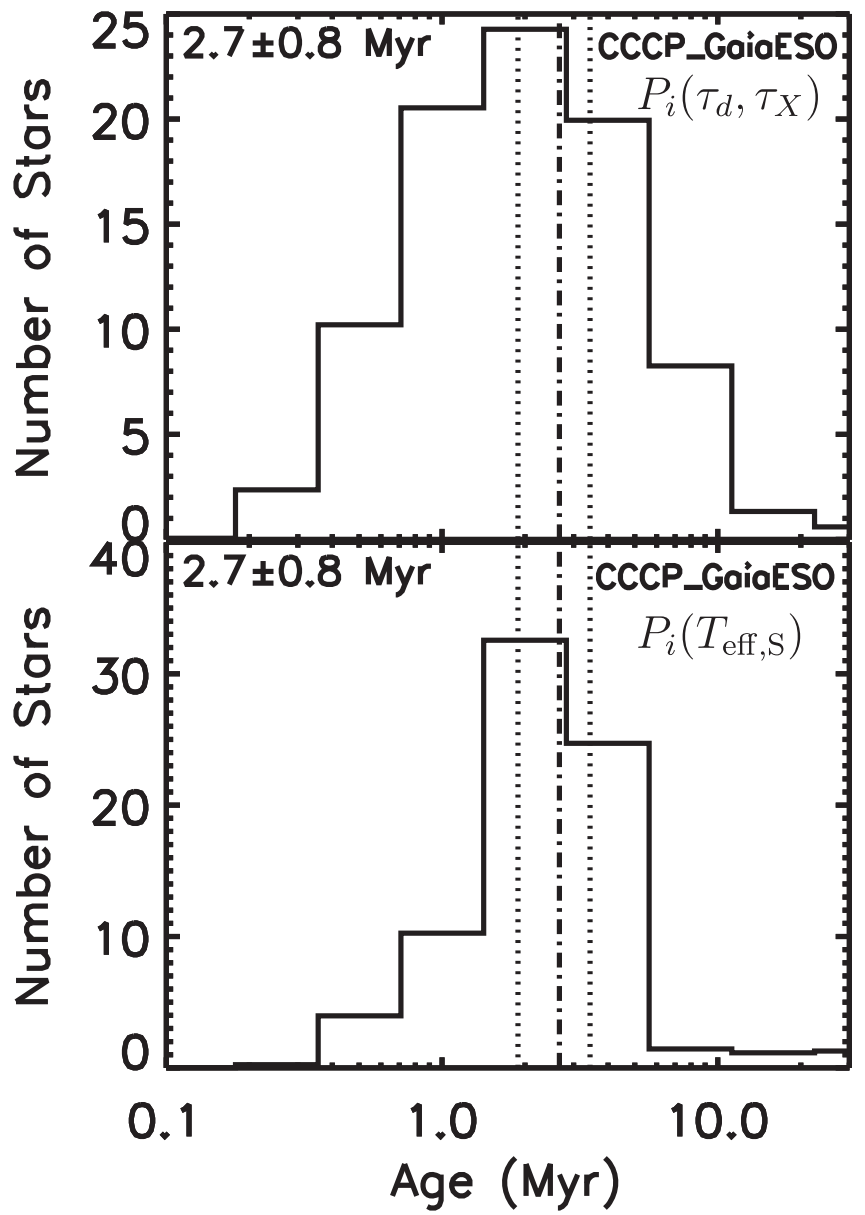

Figure 10. Age $\left(t_{\star}\right)$ distributions for all SED models fit to the spectroscopic comparison sample. The panels correspond to the pHRDs shown in Figure 9: standard weighting (top) and spectroscopic temperature constraints (bottom). In each plot, $\tau_{\mathrm{SF}}$ and its uncertainty are annotated and indicated by vertical dashed-dotted and dashed lines.

We can piece together a global star formation history of the CNC (Figure 17). The first stars formed 7-10 Myr ago as a GMC with initial mass likely exceeding $10^{6} M_{\odot}$ (Grabelsky et al. 1988; Preibisch et al. 2012). Extrapolating from the remnant morphology apparent in IR dust maps, the structure of the original GMC was probably dominated by one large filament running from southeast to northwest with one or more intersecting, smaller filaments. The initial collapse phase produced, within a few megayears, $\operatorname{Tr} 15$, multiple smaller clusters (including Bochum 11), and a distributed stellar population formed from lower-density regions that were never gravitationally bound.

The most spectacular collapse phase commenced $<3 \mathrm{Myr}$ ago, as dense gas channeled along the filaments to their main intersection nodes in the center of the nebula rapidly collapsed to form the massive $\operatorname{Tr} 16^{11}$ and 14 clusters (as well as Collinder $232^{12}$ ) in rapid succession. Feedback from the radiation and stellar winds of the very massive stars formed

\footnotetext{
${ }^{11} \operatorname{Tr} 16$ is in reality a collection of smaller subclusters (Feigelson et al. 2011; Kuhn et al. 2014; Buckner et al. 2019) that are approximately coeval (Getman et al. 2014).

12 Previous studies at visual wavelengths (Tapia et al. 2003; Hur et al. 2012) questioned the existence of the Collinder 232 cluster, probably because parts of it are heavily obscured by a dust pillar. Collinder 232 presents distinct overdensities in both X-ray sources and intermediate-mass YSOs (see Figure 4).
} 


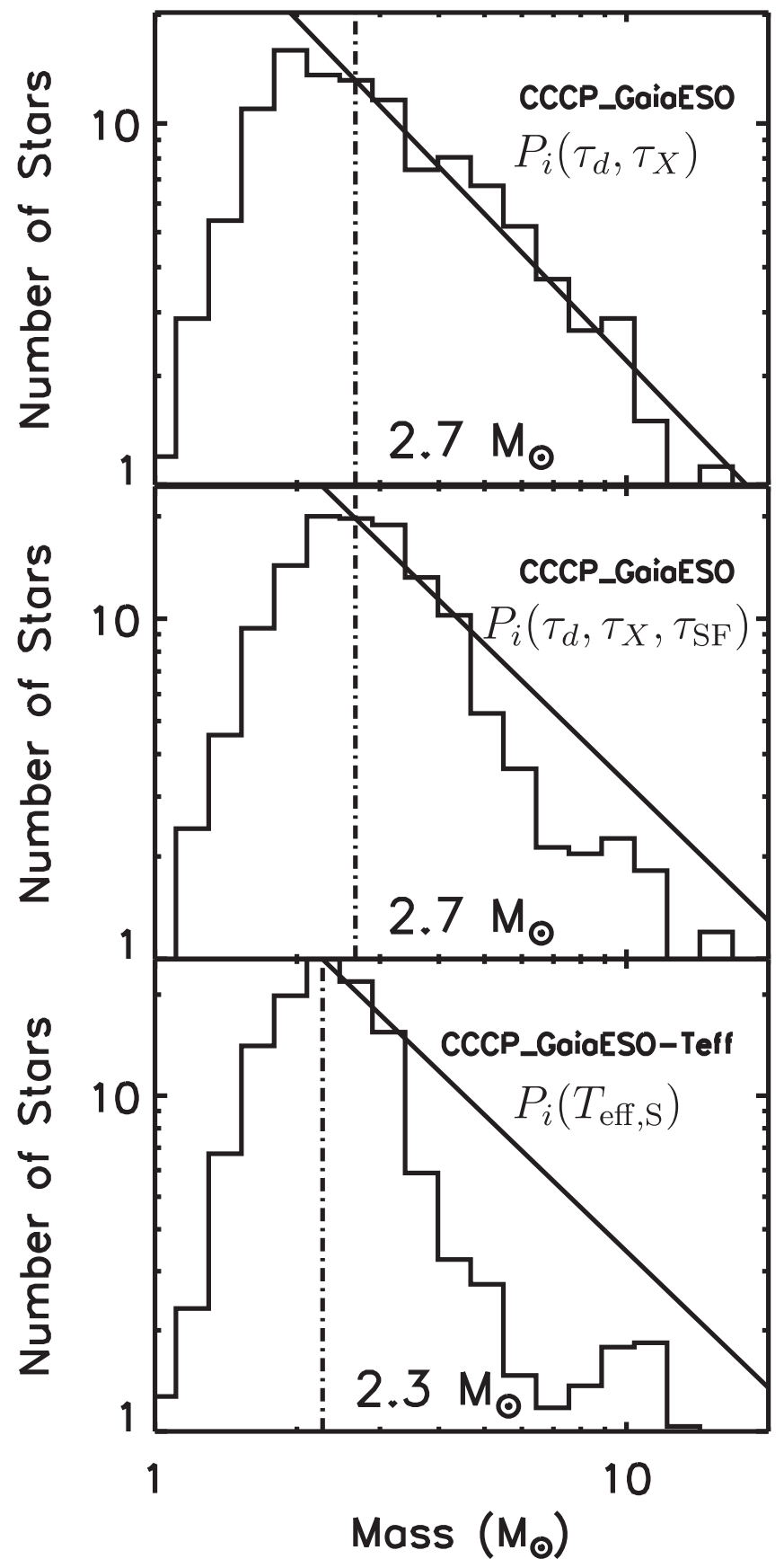

Figure 11. Mass $\left(M_{\star}\right)$ distributions for all SED models fit to the spectroscopic comparison sample using three different weighting options. Top: standard weighting (corresponding to the top panels of Figures 9 and 10). Center: isochronal weighting (Equation (6)) using $\tau_{\mathrm{SF}}=2.7 \pm 0.8 \mathrm{Myr}$. Bottom: spectroscopic temperature constraints (corresponding to the bottom panels of Figures 9 and 10). In each plot, solid lines show the Salpeter power-law slope scaled to the distribution at the cutoff mass $M_{C}$ (annotated and indicated by vertical dashed-dotted lines).

in this event (including $\eta$ Car in $\operatorname{Tr} 16$ and the O3.5-4 V((f+)) stars in Tr 14; Walborn et al. 2002; Smith \& Brooks 2007), and possibly the onset of supernovae (Townsley et al. 2011a, 2011b; Wang et al. 2011) from the most massive stars formed in the initial collapse phase sculpted the remaining molecular material, producing the global, bipolar superbubble structure of the Carina Nebula. The remaining molecular filaments were eroded, forming complex structures in the South Pillars and several pillars in the northern regions of the nebula
(Hartigan et al. 2015). The remnants of the original filamentnode structure are apparent in the large, $\mathrm{V}$-shaped dust lane immediately south of $\operatorname{Tr} 16$ and 14, and the Great Pillar farther south that points almost precisely toward the vertex of the $\mathrm{V}$ (Smith \& Brooks 2008).

The older, distributed population is consistent with dynamical evolution of many smaller subclusters over severalmegayear timescales, perhaps accelerated by the evaporation of dense gas by massive star feedback. The current, and likely final, phase of star formation in the CNC is underway within the clumpy molecular remnants, possibly triggered by massive star feedback and revealed by very young clusters of YSOs revealed by the retreat of the remaining pillars (Smith et al. 2010). In the near future, multiple supernova explosions from $\operatorname{Tr} 16$ and 14 may remove the remaining dense gas, destroying the Carina Nebula and halting star formation for good.

Previous studies of the star formation history in the CNC using observations at visual wavelengths (e.g., DeGioiaEastwood et al. 2001) were necessarily restricted to just the central, lightly obscured region containing $\operatorname{Tr} 14,15$, and 16 . But one recent study leveraged CCCP X-ray and NIR data sets to measure stellar ages across a much wider field, probing the more reddened populations. Introducing a new technique called AgeJX, Getman et al. (2014) used a combination of CCCP X-ray data and deep Very Large Telescope/HAWK-I $J H K_{S}$ photometry (Preibisch et al. 2011) to derive median isochronal ages for each of 19 small subclusters identified by Kuhn et al. (2014) plus the unclustered population. The Getman et al. (2014) study of the CNC was therefore restricted to the smaller HAWK-I field that covered the central $25 \%$ of the $1.4 \mathrm{deg}^{2}$ CCCP survey area. AgeJX is complementary to our methodology in many respects. It uses the same Chandra/ACIS X-ray point-source data, assuming an X-ray spectral shape characteristic of low-mass $\mathrm{T}$ Tauri stars to assign a mass to each star using an empirical $L_{X}-M_{\star}$ relation (Preibisch et al. 2005). An extinction-corrected $M_{J}$ is then used to place stars on the same Siess et al. (2000) pre-MS isochrones used in our naked SED models. AgeJX was restricted to ages $<5 \mathrm{Myr}$ and low-mass $\left(M_{\star}<1.2 M_{\odot}\right)$ stars, requiring deep $J$-band counterpart photometry for faint X-ray sources that may additionally lie behind large absorbing columns. The overlap between individual AgeJX sources and our CCCP IR-bright diskless sample is minimal.

The number of stars per subcluster used in the AgeJX analysis of the $\mathrm{CNC}$ ranged from 6 to 111, with typical clusters containing 20-40 stars suitable for AgeJX dating. Most of the Kuhn et al. (2014) subclusters are too small to contain a statistically robust sample of intermediate-mass stars, but each belongs to one of the larger-scale spatial overdensities (Feigelson et al. 2011) analyzed here. Specifically, region A1 ( $\operatorname{Tr} 15$ and environs) contains subclusters $\mathrm{G}, \mathrm{F}, \mathrm{H}$, and I (AgeJX 2.8-4.8 Myr); Region A2 (Tr 14 and Collinder 232) contains subclusters A-D (AgeJX 1.5-2.8 Myr); Region B (Tr 16) contains subclusters E, J, K, and L (AgeJX 2.4-3.6 Myr); and Region C (South Pillars) contains subclusters M, O, P, Q, $\mathrm{R}, \mathrm{S}$, and T (AgeJX 1.1-4.3 Myr). The upper bounds of these AgeJX ranges agree very well with our $\tau_{\mathrm{SF}}$ for the $\operatorname{Tr} 14$ and South Pillars regions (Table 2). The oldest AgeJX measurement in $\operatorname{Tr} 16$ is $3.6 \pm 0.7 \mathrm{Myr}$ for subcluster $\mathrm{K}$, which is marginally greater than $\tau_{\mathrm{SF}}$. Subcluster K contains $\eta$ Car and is therefore absent from our analysis, due to MIR saturation, but we find good agreement between AgeJX and $\tau_{\mathrm{SF}}$ for the remaining subclusters in $\operatorname{Tr} 16$. The truncation of AgeJX at $5 \mathrm{Myr}$ will 
Table 2

Duration of Star Formation in the Various CCCP Subpopulations

\begin{tabular}{|c|c|c|c|c|c|c|c|}
\hline Region/sample & $N$ & $N_{t \star}$ & $\tau_{\mathrm{SF}}(\mathrm{Myr})$ & $M_{C}\left(M_{\odot}\right)$ & $N_{C}$ & $N_{\text {IM }}$ & $f_{\mathrm{XIM}}$ \\
\hline Gaia-ESO & 139 & 88 & $2.7 \pm 0.8$ & 2.7 & 88 & 145 & 0.94 \\
\hline $\operatorname{Tr} 14 / \operatorname{Cr} 232$ & 356 & 194 & $2.6 \pm 0.5$ & 3.0 & 124 & 353 & 0.85 \\
\hline $\operatorname{Tr} 16$ & $290^{\mathrm{b}}$ & 144 & $2.6 \pm 0.5$ & 3.0 & $85^{\mathrm{b}}$ & $280^{\mathrm{b}}$ & 0.80 \\
\hline South Pillars & 319 & 156 & $4.1 \pm 0.8$ & 2.0 & 191 & 297 & 0.81 \\
\hline All & 2269 & 1168 & $3.6 \pm 0.6$ & 1.8 & 1651 & 2118 & 0.77 \\
\hline
\end{tabular}

Notes. $N$ is the total number of stars in the region/sample, and $N_{t \star}$ gives the number of model stars included in the age distribution used to determine $\tau_{\mathrm{SF}} . M_{C}$ is the mass cutoff where the second iteration of the modeled mass functions turns over from a Salpeter slope, and $N_{C}$ is the equivalent number of stars more massive than this turnover mass. $N_{\mathrm{IM}}$ gives the number of stars with $M_{\star} \geqslant 1.8 M_{\odot}$ predicted by scaling a Kroupa IMF to the final mass function, and $f_{\mathrm{XIM}}$ is the fraction of $N_{\mathrm{IM}}$ stars that were detected in X-rays.

a This sample includes 14 early-type stars with no $T_{\text {eff,S }}$ reported by D17, which we modeled using only our $W_{i}\left(\chi^{2}\right)$ weighting function.

${ }^{\mathrm{b}}$ The $\operatorname{Tr} 16$ region sample size and inferred stellar population have been significantly reduced by the photometric zone of avoidance around $\eta$ Car.

cause this method to underestimate the true ages of $\operatorname{Tr} 15$ and the distributed population. In the latter case, AgeJX returns an age of $4.0 \mathrm{Myr}$ based on 354 stars, compared to $\tau_{\mathrm{SF}}=7.2 \pm$ 2.3 Myr from 1050 stars (Table 2). Part of this discrepancy may be due to the restriction of AgeJX to the central regions of the CCCP survey area, where even stars found to be unclustered may be younger on average than those in the farther reaches of the distributed population.

We conclude that our results are broadly consistent with past estimates of stellar ages in the CNC. Nearly all of the discrepancies previously reported in the literature can be attributed to difficulties in defining membership of individual clusters, a task made even more challenging by the ubiquitous presence of the large and systematically older, distributed young stellar population.

\subsection{Impact of the Choice of Pre-MS Evolutionary Models}

A number of systematics, in particular neglect of stellar accretion histories and treating binary systems as single stars, both of which we have done here, are known to contribute to apparent isochronal age spreads of $\sim 10 \mathrm{Myr}$ on HRDs of very young $(<20 \mathrm{Myr})$ star-forming regions (see Soderblom et al. 2014 for a thorough review). That said, we can be reasonably confident in relative measurements of isochronal ages among different stellar subpopulations, and the observed intrinsic age spread across the global CNC stellar population, with its numerous subclusters, is real. The accuracy of absolute isochronal ages, however, is a matter of ongoing investigation and debate. In many evolutionary models, including those used in this study, a single isochrone fails to connect intermediatemass stars to subsolar-mass pre-MS stars in the same population, predicting younger ages for lower-mass stars (see, e.g., Hillenbrand \& White 2004; David et al. 2019).

Relatively few pre-MS evolutionary models provide detailed calculations for the $>2 M_{\odot}$ stars that dominate our CCCP IRbright sample (most exclude this mass range altogether to focus on low-mass pre-MS evolution). David et al. (2019) compare the results of placing the three most massive stellar components of eclipsing binary systems $\left(1.4,2.6\right.$, and $5.6 M_{\odot}$, respectively) in the Upper Scorpius OB Association on isochrones from the Dartmouth (Dotter et al. 2008), MIST (Choi et al. 2016; Dotter 2016), and PARSEC (v1.0; Bressan et al. 2012) evolutionary models. These authors reported good agreement among all isochrones for the ages of these intermediate-mass stars, and while the older Siess et al. (2000) isochrones were not considered, the shapes and locations of their pre-MS isochrones are very similar to the three newer model sets analyzed by David et al. (2019) across the 2-6 $M_{\odot}$ mass range.

The pre-MS isochrones and evolutionary tracks adopted in our naked SED models (Bernasconi \& Maeder 1996; Siess et al. 2000), in spite of their advanced age, still give reasonable results for intermediate-mass stars. The most promising new grid of evolutionary models appears to be the recent extension of the Geneva evolutionary tracks to the pre-MS phase by Haemmerlé et al. (2019). These models include a detailed treatment of accretion history and computation of the stellar birth line across the entire range of present-day stellar masses. They predict that stars of $<3 M_{\odot}$ are fully convective when accretion ends, which is precisely what we have observed with our large sample of X-ray-bright, diskless $2-3 M_{\odot}$ IMPS in the $\mathrm{CNC}$. The locations and shapes of the evolutionary tracks and isochrones for $t_{\star} \geqslant 1 \mathrm{Myr}$ as well as the ZAMS arrival times reported by Haemmerlé et al. (2019) for $2-5 M_{\odot}$ stars are very similar to those implemented in our models.

Some recent models for low-mass pre-MS evolution introduce the effects of magnetic fields (magnetic pressure support or large starspot covering fractions) that inhibit the contraction of fully convective stars on Hayashi tracks, increasing the isochronal ages of $<1 M_{\odot}$ pre-MS stars by factors of $\sim 2$ (Feiden et al. 2015; Somers \& Pinsonneault 2015; Feiden 2016; Jeffries et al. 2017). Intrinsic, magnetocoronal $\mathrm{X}$-ray emission characteristic of the cool, convective IMPS in our sample (E. H. Nuñez et al. 2019, in preparation) raises the question of whether a similar correction for isochronal ages of IMPSs might be warranted. We suspect any such correction would be small, however, because a full factor of 2 increase in our reported ages would place the birth of the massive $\operatorname{Tr} 14$ and 16 stellar clusters at 5-6 Myr ago, which exceeds the lifetimes of their most massive stars. This would, in turn, require the massive stars in $\operatorname{Tr} 16$ and $\operatorname{Tr} 14$ to form $\gtrsim 2 \mathrm{Myr}$ later than the intermediate-mass stars in the same (sub)clusters.

Uncertainties in pre-MS isochronal ages provide the most important systematic uncertainty in our results, but based on our review of the current literature, this systematic appears to 

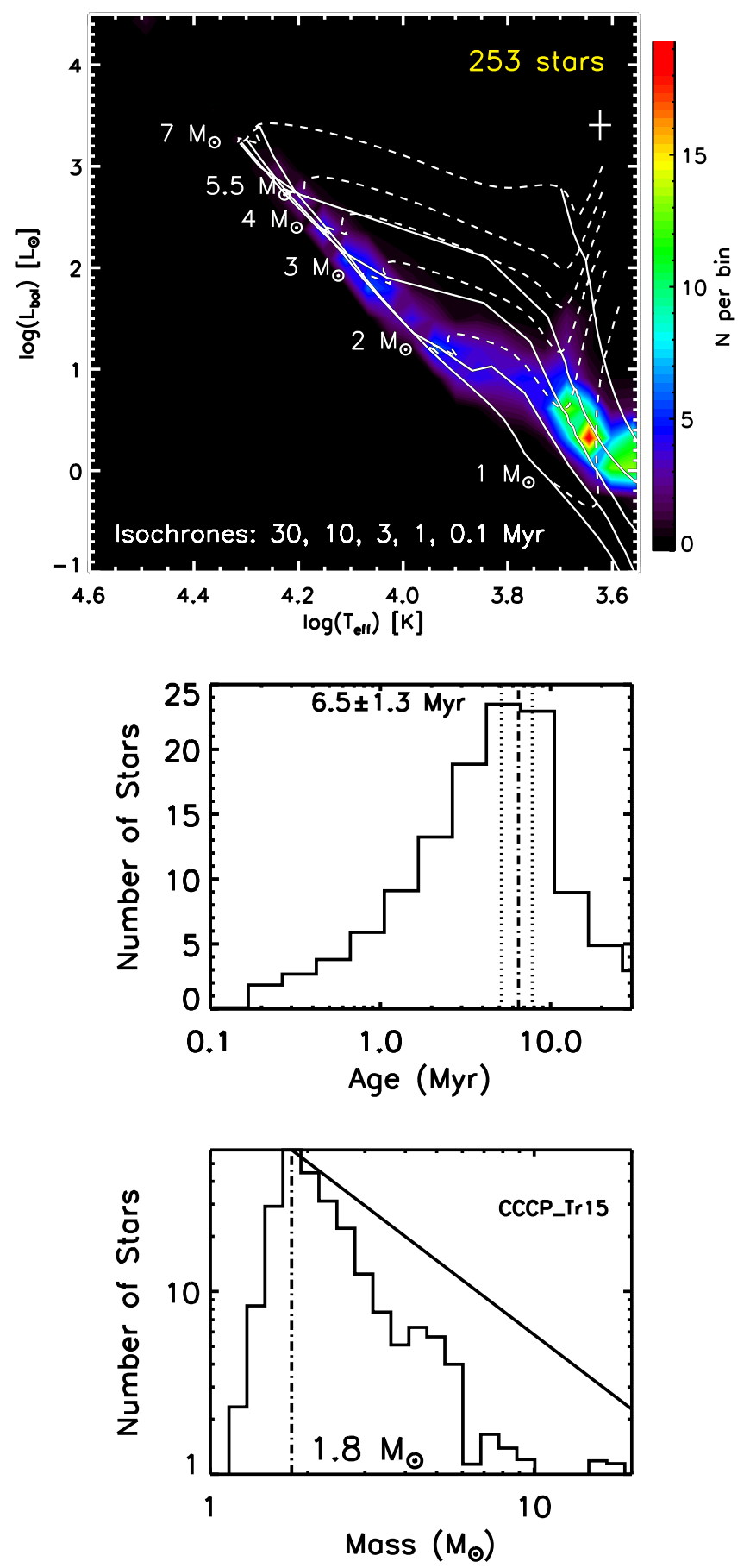

Figure 12. Top to bottom: pHRD, $t_{\star}$, and $M_{\star}$ distributions for CCCP Region A1: $\operatorname{Tr} 15$ and environs.

be considerably smaller than the factor of $\sim 2$ differences between the magnetic and nonmagnetic low-mass isochrones.

\subsection{IMPS and the "X-Ray Desert"}

Nuñez et al. (2019, in preparation) present a spectral fitting analysis of the several hundred brightest CCCP X-ray sources (excluding OB stars) and found that those with IR counterparts classified as IMPS on the pHRD were systematically more luminous in X-rays compared to lower-mass $\mathrm{T}$ Tauri stars or intermediate-mass AB stars. IMPS extend the convective $\mathrm{T}$ Tauri star relation of $L_{X} / L_{\mathrm{bol}} \sim 10^{-3.6}$ (Preibisch et al. 2005) to
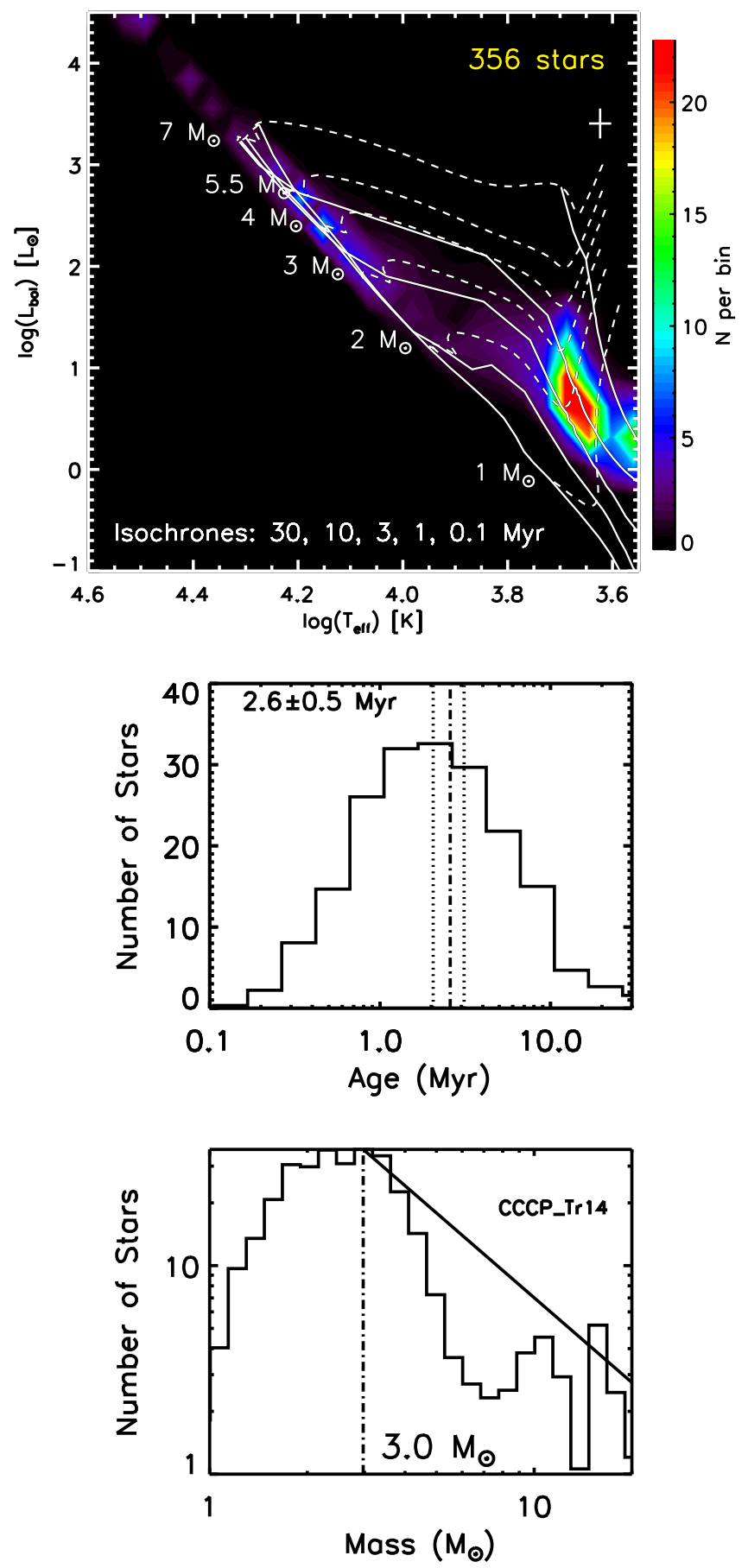

Figure 13. Top to bottom: pHRD, $t_{\star}$, and $M_{\star}$ distributions for CCCP Region A2: $\operatorname{Tr} 14$, Collinder 232, and environs.

higher X-ray luminosities $\left(L_{X} \sim 10^{32} \mathrm{erg} \mathrm{s}^{-1}\right)$. The strongest stellar coronal X-ray flares may thus be produced by IMPS. However, after IMPS complete their descent of the convective Hayashi tracks, they develop radiative cores that grow rapidly, sending stars horizontally across Henyey tracks toward the ZAMS (producing the above-mentioned R-C gap described by Mayne et al. 2007, which is apparent on all of our pHRDs but most pronounced for $\operatorname{Tr} 14$ and 16; see Figures 13 and 14, respectively). The growth of the radiative core drastically alters the magnetic field structure of the star (Gregory et al. 2012). Unlike the case for low-mass stars, the IMPS dynamo-driven 

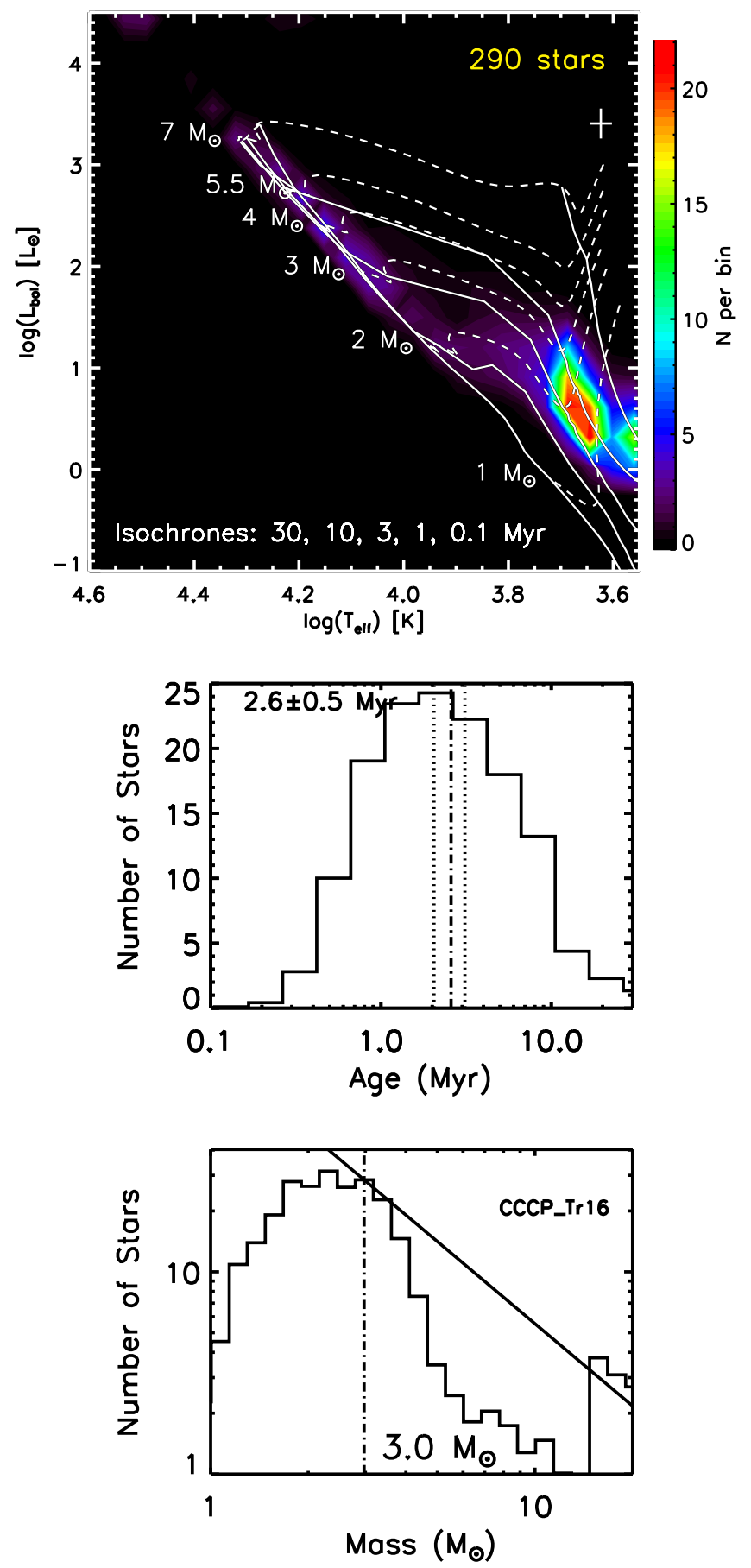

Figure 14. Top to bottom: pHRD, $t_{\star}$, and $M_{\star}$ distributions for CCCP Region B: $\operatorname{Tr} 16$ and environs.

X-ray emission decays rapidly after the R-C transition (Gregory et al. 2016) and should disappear completely prior to arrival on the fully radiative ZAMS.

Because a greater fraction of intermediate-mass stars reach the ZAMS and become X-ray dark as a population ages, we expect that the magnitude of this deficit, (the "aridity" of the X-ray desert) will increase as a function of $\tau_{\mathrm{SF} .}{ }^{13}$ To explore this effect, for the mass distribution of each region or sample in

\footnotetext{
13 For the youngest stellar populations, we expect a mass distribution consistent with Salpeter for X-ray-selected, diskless IMPSs, as observed by P16 for the IR-dark cloud M17 SWex.
}
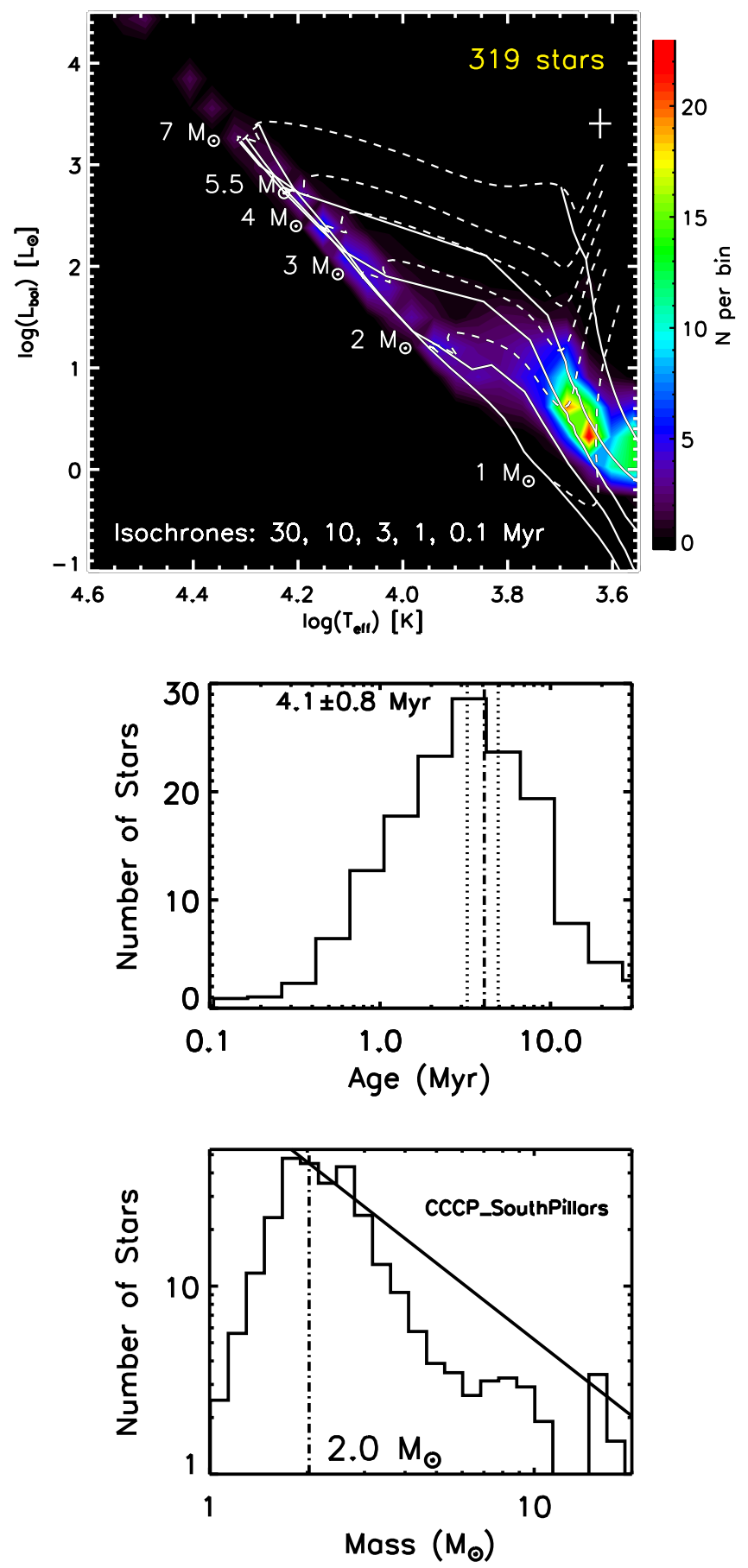

Figure 15. Top to bottom: pHRD, $t_{\star}$, and $M_{\star}$ distributions for CCCP Region C, the South Pillars.

Table 2, we define a parameter $f_{\mathrm{XIM}}$, the fraction of X-raydetected intermediate-mass stars with $M_{\star} \geqslant 1.8 M_{\odot}$. We estimate the expected number $N_{\text {IM }}$ of intermediate-mass stars in the parent population by integrating the Salpeter IMF (scaled to the value of the $M_{\star}$ distribution at the cutoff mass $M_{C}$ ). After correcting for the varying mass incompleteness in each $M_{\star}$ distribution in the range $1.8 M_{\odot} \leqslant M_{\star}<M_{C}$ using the same scaled Salpeter IMF, we integrate over this corrected distribution to find $f_{\text {XIM }}$.

As expected, we observe a trend of decreasing $f_{\mathrm{XIM}}$ with increasing $\tau_{\mathrm{SF}}$ (Figure 18; error bars on $f_{\mathrm{XIM}}$ are estimated assuming Poisson counting statistics for each bin in the $M_{\star}$ 

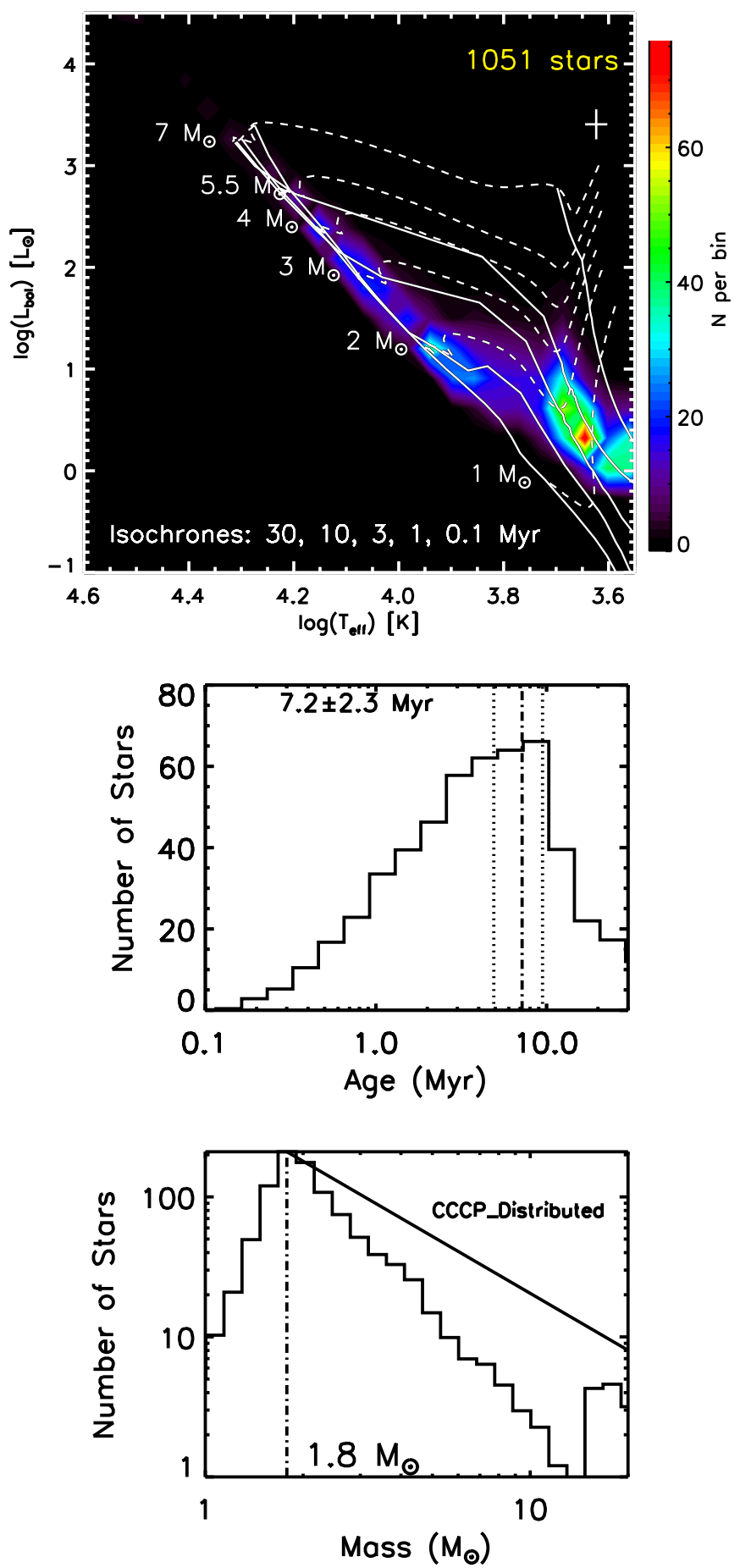

Figure 16. Top to bottom: pHRD, $t_{\star}$, and $M_{\star}$ distributions for CCCP Region D, the distributed population.

distribution). It would be tempting to use this qualitative trend to infer the real binary fraction among intermediate-mass stars, for example, but systematic effects preclude us from drawing such firm, quantitative conclusions. One complication is that $N_{\text {IM }}$ provides only a lower limit on the true stellar population, because many intermediate-mass stars in the CNC still have disks and were hence not counted among our sample. Furthermore, we have no information about the frequency at which real binary companions are detected as X-ray point sources, the sensitivity of which varies across the large CCCP
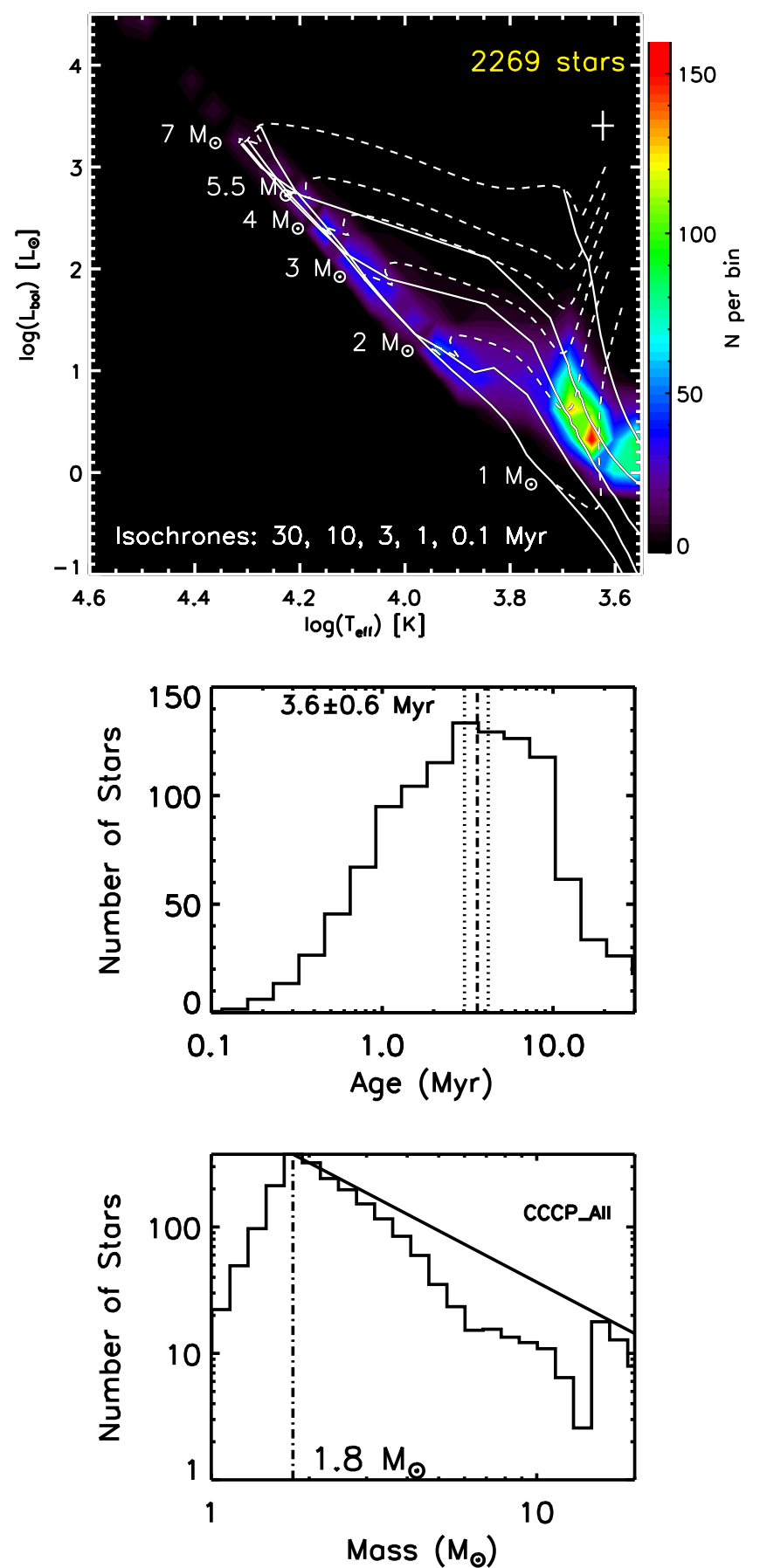

Figure 17. Top to bottom: pHRD, $t_{\star}$, and $M_{\star}$ distributions for all CCCP X-rayselected, diskless members with valid SED fits. To produce the mass function shown here, no single isochronal age value was appropriate, so we instead aggregated the $M_{\star}$ distributions from each constituent subregion (Figures 12-16).

mosaic (Broos et al. 2011b). But the systematic most relevant to the current analysis involves the shape of the $M_{\star}$ distributions themselves, given the challenges of precisely constraining stellar masses from SED modeling. For the GaiaESO spectroscopic comparison sample, we find a deeper deficit in intermediate-mass stars with the more stringent $P\left(T_{\text {eff,S }}\right)$ constraints on the SED models than with our standard $P\left(\tau_{d}, \tau_{x}\right)$ weighting $\left(f_{\mathrm{XIM}}=0.78\right.$ versus 0.94 ; see Figure 11 and Table 2). This is purely an artifact of limited precision in measuring stellar masses via broadband photometry alone. A 


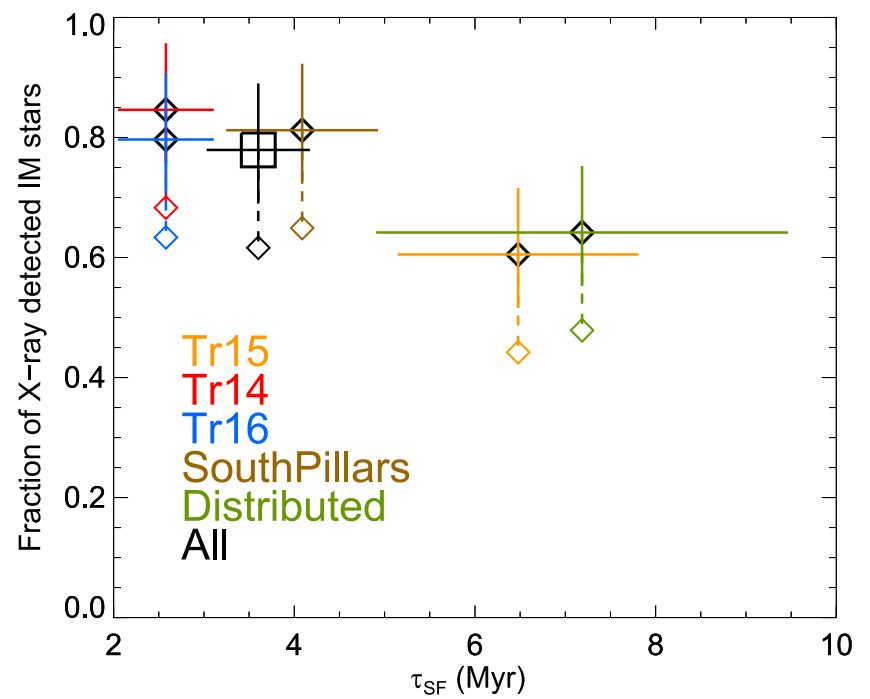

Figure 18. Trend of decreasing $f_{\mathrm{XIM}}$ with increasing $\tau_{\mathrm{SF}}$. The dashed lines and the colored diamonds beneath them show the correction for the systematic offset introduced by the intrinsic spread in the modeled mass distributions.

tentative correction for this effect, simply $f_{\mathrm{XIM}}-0.16$, is also illustrated in Figure 18. We caution that this correction is not strictly appropriate, given the additional selection criteria imposed by D17 and by us on the Gaia-ESO comparison sample that were not imposed on the full CCCP IR-bright sample, but it does extend the uncertainty on $f_{\mathrm{XIM}}$ to encompass a more plausible range of values.

\section{Conclusions}

We have presented a pilot study in which we have developed a novel methodology for constraining the duration of star formation and applied it to multiple young stellar populations observed in the Carina Nebula Complex. We employ the results of IR SED fitting to individual X-ray-selected, diskless intermediate-mass stars to synthesize models of the ensemble population and probe distributions of stellar masses and ages. Because IMPS rapidly evolve along radiative tracks to become late B- and A-type stars on the ZAMS (including Herbig $\mathrm{Ae} / \mathrm{Be}$ stars), they serve as the most sensitive available chronometers for the first $\sim 10 \mathrm{Myr}$ in the evolution of massive stellar clusters and associations.

Our SED modeling methodology uses all available photometric data simultaneously, incorporating external age constraints based on the lack of mid-IR excess emission and the presence of X-ray emission that are communicated to the model via weighting functions. We treat each star as a cloud of probability in SED model parameter space, analyzing the $\log L_{\mathrm{bol}}-\log T_{\text {eff }}$ plane (pHRD) coupled with mass and age distributions. This avoids the pitfalls of interpreting individual photometry data points with Gaussian error bars, which can be large and are inherently asymmetric with respect to nonlinear isochrones and evolutionary tracks. We validate our technique using a comparison sample of 139 stars with spectroscopic $T_{\text {eff,S }}$ measurements or broad constraints from the Gaia-ESO survey (D17), and we find that we can successfully identify the correct isochronal age from the weighted SED models fit to broadband IR photometry alone. This validation exercise provides a road map for what we would consider a "gold standard" for observational studies of young stellar populations in massive Galactic star-forming regions suffering from severe differential extinction: visual/NIR spectroscopy to measure $T_{\text {eff,S }}$ for individual stellar sources combined with IR SED modeling analysis to tightly constrain $L_{\mathrm{bol}}$ and reddening.

Our study encompasses the entire $1.4 \mathrm{deg}^{2}$ field of the CCCP, including the substantial fraction of distributed or significantly reddened stars in the $\mathrm{CNC}$, providing a homogeneous analysis of wide-field X-ray and IR imaging data. We find that star formation commenced throughout the CNC $\sim 10 \mathrm{Myr}$ in the past (as proposed by DeGioia-Eastwood et al. 2001), after which the SFR rapidly accelerated to produce the massive Tr 15 cluster $6.5 \mathrm{Myr}$ ago and peaked 2-3 Myr ago with the birth of the very massive Tr 16 and 14 clusters, which contain some of the most massive stars known in the Galaxy. Our results generally agree with isochronal ages reported previously for various constituent CNC (sub)clusters (e.g., Feinstein et al. 1980; Carraro 2002; Tapia et al. 2003; Ascenso et al. 2007; Hur et al. 2012; Getman et al. 2014).

Our X-ray selection criterion imposes unique selection biases on the resultant stellar samples. In particular, the powerful coronal X-ray emission produced by convective IMPS disappears once these objects reach the ZAMS along radiative tracks (Gregory et al. 2016, Nuñez et al. 2019, in preparation). We observe the effects of this selection bias as a deficit of intermediate-mass stars in our modeled mass distributions with respect to a standard Salpeter IMF slope, the magnitude of which increases with age of the stellar population. Late B- and A-type stars (including Herbig Ae/Be stars) near the ZAMS can also be included in our samples if they have lower-mass, convective stellar companions producing detectable X-ray emission.

Subsequent papers in this series will implement our methodology for a uniform comparative study of the duration of star formation in more than two dozen other Galactic massive star-forming regions for which comparable X-ray and IR photometric data exist. The results of this extended study will enable improved measurements of SFRs, star-forming efficiencies, and the dynamical evolution of giant $\mathrm{H}$ II regions.

We thank M.A. Kuhn and L.A. Hillenbrand for numerous discussions and suggestions that substantially improved this work. We are grateful to the anonymous referee for helpful comments and suggestions that improved the interpretation of the spectroscopic comparison sample and the explanation of our methodology. This research was supported by the NSF through grant CAREER-1454333 (PI M.S. Povich). J.T.M. and E.H.N. acknowledge support from the Cal-Bridge program through NSF awards DUE-1356133 and AST-1559559. The scientific results are based in part on observations made by the Chandra X-ray Observatory and published previously in cited articles. This work is based in part on archival data obtained with the Spitzer Space Telescope, which is operated by the Jet Propulsion Laboratory, California Institute of Technology under a contract with NASA. This publication makes use of data products from the Two Micron All-Sky Survey, which is a joint project of the University of Massachusetts and the Infrared Processing and Analysis Center/California Institute of Technology, funded by NASA and the NSF.

Facilities: CXO (ACIS), Spitzer (IRAC), CTIO:2MASS.

\section{ORCID iDs}

Matthew S. Povich (1) https://orcid.org/0000-0001-9062-3583 Thomas P. Robitaille (ib https://orcid.org/0000-0002-

8642-1329 


\section{References}

Arthur, S. J., Henney, W. J., Mellema, G., de Colle, F., \& Vázquez-Semadeni, E. 2011, MNRAS, 414, 1747

Ascenso, J., Alves, J., Vicente, S., \& Lago, M. T. V. T. 2007, A\&A, 476, 199 Benjamin, R. A., Churchwell, E., Babler, B. L., et al. 2003, PASP, 115, 953 Bernasconi, P. A., \& Maeder, A. 1996, A\&A, 307, 829

Bressan, A., Marigo, P., Girardi, L., et al. 2012, MNRAS, 427, 127

Broos, P. S., Getman, K. V., Povich, M. S., et al. 2011a, ApJS, 194, 4

Broos, P. S., Townsley, L. K., Feigelson, E. D., et al. 2011b, ApJS, 194, 2

Buckner, A. S. M., Khorrami, Z., Khalaj, P., et al. 2019, A\&A, 622, A184

Burkhart, B. 2018, ApJ, 863, 118

Calvet, N., Muzerolle, J., Briceño, C., et al. 2004, AJ, 128, 1294

Carraro, G. 2002, MNRAS, 331, 785

Castelli, F., \& Kurucz, R. L. 2004, arXiv:astro-ph/0405087

Choi, J., Dotter, A., Conroy, C., et al. 2016, ApJ, 823, 102

Chomiuk, L., \& Povich, M. S. 2011, AJ, 142, 197

Churchwell, E., Babler, B. L., Meade, M. R., et al. 2009, PASP, 121, 213

Damiani, F., Klutsch, A., Jeffries, R. D., et al. 2017, A\&A, 603, A81 (D17)

David, T. J., Hillenbrand, L. A., Gillen, E., et al. 2019, ApJ, 872, 161

DeGioia-Eastwood, K., Throop, H., Walker, G., \& Cudworth, K. M. 2001, ApJ, 549, 578

Dotter, A. 2016, ApJS, 222, 8

Dotter, A., Chaboyer, B., Jevremović, D., et al. 2008, ApJS, 178, 89

Evans, N. R., DeGioia-Eastwood, K., Gagné, M., et al. 2011, ApJS, 194, 13

Fall, S. M., Chandar, R., \& Whitmore, B. C. 2005, ApJL, 631, L133

Fazio, G. G., Hora, J. L., Allen, L. E., et al. 2004, ApJS, 154, 10

Feiden, G. A. 2016, A\&A, 593, A99

Feiden, G. A., Jones, J., \& Chaboyer, B. 2015, in 18th Cambridge Workshop on Cool Stars, Stellar Systems, and the Sun, ed. H. C. Harris \& G. van Belle, 171

Feigelson, E. D., Getman, K. V., Townsley, L. K., et al. 2011, ApJS, 194, 9

Feigelson, E. D., Townsley, L. K., Broos, P. S., et al. 2013, ApJS, 209, 26

Feinstein, A., Fitzgerald, M. P., \& Moffat, A. F. J. 1980, AJ, 85, 708

Figer, D. F., Kim, S. S., Morris, M., et al. 1999, ApJ, 525, 750

Gagné, M., Fehon, G., Savoy, M. R., et al. 2011, ApJS, 194, 5

Gaia Collaboration 2018, A\&A, 616, A1

Getman, K. V., Feigelson, E. D., Kuhn, M. A., et al. 2014, ApJ, 787, 108

Gilmore, G., Randich, S., Asplund, M., et al. 2012, Msngr, 147, 25

Grabelsky, D. A., Cohen, R. S., Bronfman, L., \& Thaddeus, P. 1988, ApJ, 331,181

Gregory, S. G., Adams, F. C., \& Davies, C. L. 2016, MNRAS, 457, 3836

Gregory, S. G., Donati, J.-F., Morin, J., et al. 2012, ApJ, 755, 97

Haemmerlé, L., Eggenberger, P., Ekström, S., et al. 2019, A\&A, 624, A137

Hartigan, P., Reiter, M., Smith, N., \& Bally, J. 2015, AJ, 149, 101

Herbst, W., \& Shevchenko, V. S. 1999, AJ, 118, 1043

Hernández, J., Hartmann, L., Megeath, T., et al. 2007, ApJ, 662, 1067

Hillenbrand, L. A., Strom, S. E., Vrba, F. J., \& Keene, J. 1992, ApJ, 397, 613

Hillenbrand, L. A., \& White, R. J. 2004, ApJ, 604, 741

Hopkins, P. F., Kereš, D., Oñorbe, J., et al. 2014, MNRAS, 445, 581

Hopkins, P. F., Wetzel, A., Kereš, D., et al. 2018, MNRAS, 480, 800

Hur, H., Sung, H., \& Bessell, M. S. 2012, AJ, 143, 41

Indebetouw, R., Mathis, J. S., Babler, B. L., et al. 2005, ApJ, 619, 931

Jeffries, R. D., Jackson, R. J., Franciosini, E., et al. 2017, MNRAS, 464, 1456

Kennicutt, R. C., \& Evans, N. J. 2012, ARA\&A, 50, 531

Köhler, R., Kasper, M., Herbst, T. M., Ratzka, T., \& Bertrang, G. H.-M. 2016, A\&A, 587, A35

Koo, B.-C., \& McKee, C. F. 1992, ApJ, 388, 93

Kroupa, P., \& Weidner, C. 2003, ApJ, 598, 1076

Krumholz, M. R., Dekel, A., \& McKee, C. F. 2012, ApJ, 745, 69
Kuhn, M. A., Feigelson, E. D., Getman, K. V., et al. 2014, ApJ, 787, 107

Kuhn, M. A., Hillenbrand, L. A., Sills, A., Feigelson, E. D., \& Getman, K. V. 2019, ApJ, 870, 32

Lindegren, L., Hernández, J., Bombrun, A., et al. 2018, A\&A, 616, A2

Massey, P., \& Hunter, D. A. 1998, ApJ, 493, 180

Mayne, N. J., Naylor, T., Littlefair, S. P., Saunders, E. S., \& Jeffries, R. D. 2007, MNRAS, 375, 1220

McKee, C. F., \& Ostriker, E. C. 2007, ARA\&A, 45, 565

Offner, S. S. R., Clark, P. C., Hennebelle, P., et al. 2014, in Protostars and Planets VI, ed. H. Beuther (Tucson, AZ: Univ. Arizona Press), 53

Palla, F., \& Stahler, S. W. 1991, ApJ, 375, 288

Pecaut, M. J., \& Mamajek, E. E. 2013, ApJS, 208, 9

Povich, M. S., Busk, H. A., Feigelson, E. D., Townsley, L. K., \& Kuhn, M. A. 2017, ApJ, 838, 61

Povich, M. S., Kuhn, M. A., Getman, K. V., et al. 2013, ApJS, 209, 31

Povich, M. S., Smith, N., Majewski, S. R., et al. 2011a, ApJS, 194, 14

Povich, M. S., Townsley, L. K., Broos, P. S., et al. 2011b, ApJS, 194, 6

Povich, M. S., Townsley, L. K., Robitaille, T. P., et al. 2016, ApJ, 825, 125 (P16)

Preibisch, T., \& Feigelson, E. D. 2005, ApJS, 160, 390

Preibisch, T., Hodgkin, S., Irwin, M., et al. 2011, ApJS, 194, 10

Preibisch, T., Kim, Y.-C., Favata, F., et al. 2005, ApJS, 160, 401

Preibisch, T., Roccatagliata, V., Gaczkowski, B., \& Ratzka, T. 2012, A\&A, 541, A132

Robitaille, T. P. 2017, A\&A, 600, A11 (R17)

Robitaille, T. P., Whitney, B. A., Indebetouw, R., \& Wood, K. 2007, ApJS, 169,328

Robitaille, T. P., Whitney, B. A., Indebetouw, R., Wood, K., \& Denzmore, P. 2006, ApJS, 167, 256

Roccatagliata, V., Preibisch, T., Ratzka, T., \& Gaczkowski, B. 2013, A\&A, 554, A6

Salpeter, E. E. 1955, ApJ, 121, 161

Siess, L., Dufour, E., \& Forestini, M. 2000, A\&A, 358, 593

Skrutskie, M. F., Cutri, R. M., Stiening, R., et al. 2006, AJ, 131, 1163

Smith, N., \& Brooks, K. J. 2007, MNRAS, 379, 1279

Smith, N., \& Brooks, K. J. 2008, in Handbook of Star-forming Regions, Vol. II, ed. B. Reipurth (San Francisco, CA: ASP), 138

Smith, N., Povich, M. S., Whitney, B. A., et al. 2010, MNRAS, 406, 952

Smith, N., Stassun, K. G., \& Bally, J. 2005, AJ, 129, 2

Soderblom, D. R., Hillenbrand, L. A., Jeffries, R. D., Mamajek, E. E., \& Naylor, T. 2014, in Protostars and Planets VI, ed. H. Beuther et al. (Tucson, AZ: Univ. Arizona Press), 219

Somers, G., \& Pinsonneault, M. H. 2015, MNRAS, 449, 4131

Tapia, M., Roth, M., Vázquez, R. A., \& Feinstein, A. 2003, MNRAS, 339, 44

Townsley, L. K., Broos, P. S., Chu, Y.-H., et al. 2011a, ApJS, 194, 15

Townsley, L. K., Broos, P. S., Corcoran, M. F., et al. 2011b, ApJS, 194, 1

Townsley, L. K., Broos, P. S., Garmire, G. P., et al. 2014, ApJS, 213, 1

Townsley, L. K., Broos, P. S., Garmire, G. P., et al. 2018, ApJS, 235, 43

Vázquez-Semadeni, E., Gómez, G. C., Jappsen, A.-K., Ballesteros-Paredes, J., \& Klessen, R. S. 2009, ApJ, 707, 1023

Vogelsberger, M., Genel, S., Springel, V., et al. 2014, MNRAS, 444, 1518

Walborn, N. R., Howarth, I. D., Lennon, D. J., et al. 2002, AJ, 123, 2754

Wang, J., Feigelson, E. D., Townsley, L. K., et al. 2011, ApJS, 194, 11

Weaver, R., McCray, R., Castor, J., Shapiro, P., \& Moore, R. 1977, ApJ, 218,377

Wright, N. J., Drake, J. J., Guarcello, M. G., et al. 2014, arXiv:1408.6579

Zamora-Avilés, M., Vázquez-Semadeni, E., González, R. F., et al. 2019, MNRAS, 487, 2200

Zinnecker, H., \& Yorke, H. W. 2007, ARA\&A, 45, 481 